\title{
A mixture theory-based concrete corrosion model coupling chemical reactions, diffusion and mechanics
}

\author{
Arthur J. Vromans ${ }^{1,2^{*}}$, Adrian Muntean ${ }^{2}$ and Fons van de Ven ${ }^{1}$
}

\begin{abstract}
A 3-D continuum mixture model describing the corrosion of concrete with sulfuric acid is built. Essentially, the chemical reaction transforms slaked lime (calcium hydroxide) and sulfuric acid into gypsum releasing water. The model incorporates the evolution of chemical reaction, diffusion of species within the porous material and mechanical deformations. This model is applied to a 1-D problem of a plate-layer between concrete and sewer air. The influx of slaked lime from the concrete and sulfuric acid from the sewer air sustains a gypsum creating chemical reaction (sulfatation or sulfate attack). The combination of the influx of matter and the chemical reaction causes a net growth in the thickness of the gypsum layer on top of the concrete base. The model allows for the determination of the plate layer thickness $h=h(t)$ as function of time, which indicates both the amount of gypsum being created due to concrete corrosion and the amount of slaked lime and sulfuric acid in the material. The existence of a parameter regime for which the model yields a non-decreasing plate layer thickness $h(t)$ is identified numerically. The robustness of the model with respect to changes in the model parameters is also investigated.
\end{abstract}

Keywords: Reaction-diffusion, Mechanics, Mixture theory, Concrete corrosion, Sulfatation attack

Mathematics Subject Classification (2010): Primary 74D05; 74F20; secondary 74B05; 74F10; 74F25

PACS Codes: Primary 47.70.Fw; 83.10.Ff; secondary 46.70.-p; 47.10.Ab; 83.60.BC

\section{Introduction}

Forecasting concrete corrosion is a major issue in civil engineering due to its potential of drastically decreasing the lifespan of constructions such as sewers, bridges and dams, see e.g. [13, 33, 35]. As an example, the differences in mechanical properties between gypsum and concrete result in volume expansion, cracking, and decrease in load-bearing capacity of the concrete resulting in compromised structural integrity followed by expensive repairs, construction replacements or even accidents due to (partial) collapse $[19,38]$ resulting in major costs for society $[14,39]$.

We focus on three related topics: Firstly, we aim to construct a 3-D continuum mixture model describing

*Correspondence: a.j.vromans@tue.nl

${ }^{1}$ Centre for Analysis, Scientific Computing and Applications (CASA), Technische Universiteit Eindhoven, Den Dolech 2,5612AZ Eindhoven, The Netherlands 2Department of Mathematics and Computer Science, Karlstads Universitet, Universitetsgatan 2,65188 Karlstad, Sweden concrete corrosion capable of exhibiting realistic behaviour of the growth of a concrete layer due to the formation of gypsum inside the concrete layer. Secondly, we apply the new model to a specific 1-D situation of the concrete layer and investigate the validity of the behavior of this 1-D model with respect to physical constraints and expected physical behavior. Finally, we investigate the parameter dependence of both the time span of realistic behavior and growth of the concrete layer for the 1-D model.

Even though concrete is a heterogeneous material, a lot of research has been done relying on continuum models, where the heterogeneity details are averaged out. In [26] the reader can find a short historical overview of the use of continuum models in concrete research. Similar to the continuum models from [26], the authors of ref. [31] proposed a composite material model of concrete with an explicit volume division into mortar and aggregate. These models were mostly created to better 
describe the behavior of concrete under high stresses, and, hence, to predict the cracking behavior observed in the experiments reported in $[26,31]$. The mathematical community has addressed this corrosion issue mainly from a single-scale or multiple-scale reaction-diffusion perspective. Usually, the single scale approach involves one or two moving sharp reaction interfaces $[1,12,17,18,29,30]$, while the multiple scale setting prefers exploiting a better understanding of the porosity and tortuosity of the material without involving free boundaries $[2,10,16]$. There are still a number of open issues concerning on how poro-mechanics of the material couples with chemical reactions, flow, diffusion and heat transfer hindering a successful forecast of the durability of the concrete exposed to sulfate attack. In this paper, we are interested in understanding and then predicting eventual critical situations occurring before cracking. Particularly, we want to describe the corrosion of concrete by acid attack [37], which usually leads at a later stage to cracking followed by erosion. The main inspiration source for our problem setting is the basic scenario described in [4] which considers a simple reaction mechanism producing gypsum, without involving the ettringite formation.

In [4] an isothermal acid attack continuum model for sulfuric acid corrosion was proposed with a similar sewer pipe geometry as in our model, but including also the porosity of the gypsum. This model focussed solely on the creation of hydrogen sulfide and sulfuric acid, which reacts at the boundary to create gypsum. The model assumed that almost all the gypsum was created at the boundary separating the uncorroded concrete causing a moving sharp corrosion front penetrating irreversibly the material. We deviate from this model by assuming that the gypsum reaction gradually takes place in the full domain, and that the corrosion front is caused by the penetration of sulfuric acid. In some sense our model can be seen as a description of the moving corrosion front in [4] as a fixed bulk reaction domain, and can, therefore, be idealized into a plate-layer model. To avoid describing the exact growth of the involved phases of the material, we take a modeling route in the spirit of the classical mixture theory.

Figure 1 shows the concrete geometry we have in mind. A concrete sewer pipe contains in the air phase acid droplets wanting to react with the concrete to form gypsum. When zooming in the pipe wall, the curvature vanishes, which allows for the formulation of a plate layer model of the concrete pipe. We neglect, therefore, the tangential directions and only focus on the normal $(z)$ direction. Hence, a 1-D model can be posed to approximate the concrete corrosion in a simplistic 3-D sewer pipe.

It is worth noting that most of the assumptions mentioned in [4] are taken over here as well. For example, our model is supposed to reflect the entire corrosion process with no other contributing chemical reactions and species than those explicitly mentioned. Also, the external concentration and influx rates of sulfuric acid and hydrogen sulfide are constant. Both these assumptions are restrictive. For example, competing corrosion reactions and other reacting chemicals, such as nitrates, are present in an actual concrete corrosion process according to [4]. Moreover, in [9] it is explained that experiments show that external concentrations and influx rates are not even approximately constant because flow changes (changing Reynolds number) have enormous influences, which according to [4] could change rates and concentrations with many powers of 10 . Hence, the assumptions of ref. [4] are necessary to reduce the complexity of our model.

Our paper is organized as follows. In Section 2, we construct several 3-D continuum mixture models of chemical corrosion of concrete. We take into account effective balance laws, diffusion processes, chemical reaction effects, mechanical effects due to elastic and/or viscoelastic stresses, local interactions due to for instance the Stokes drag, and influx from external reservoirs and from domain growth due to a moving corrosion layer. In Section 3, we focus on the normal $(z)$ direction to obtain an effective 1-D model of the corroding concrete for one of the constructed models. In Section 4, we briefly describe both the code used to simulate the model of Section 3 and the validation of this code with respect to the asymptotic expansion solution obtained in Appendix A. In Section 5, we investigate the validity of the numerical behavior of the model of Section 3. In Subsection 5.1, we investigate the dependence of the realistic behavior on specific tuples of model parameters. Finally, in the conclusion we summarize our results and discuss the relation of these results with known literature.

\section{Derivation of a mixture-theory-based concrete corrosion model}

The presentation of a continuous 3-component mixture model in this section is based on the theory of mixtures of Bowen in [7].

\subsection{Preliminaries}

Let the index $\alpha$ denote the different constituents of our mixture, $\alpha=1$ the gypsum (solid), $\alpha=2$ the lime (solid) and $\alpha=3$ the acid (fluid). The configuration $\mathcal{G}(t)$ indicates the domain occupied by the mixture body at time $t>0$ in $\mathbf{R}_{3}$, and $\mathbf{x}=\mathbf{x}(t) \in \mathcal{G}(t)$ is the momentary position of a material point of the mixture body. Let $g(t) \subset \mathcal{G}(t)$ be a generic element of material volume, which by definition has no fixed volume. This partial material volume $g(t)$ contains $n_{\alpha}(g(t))$ molecules 


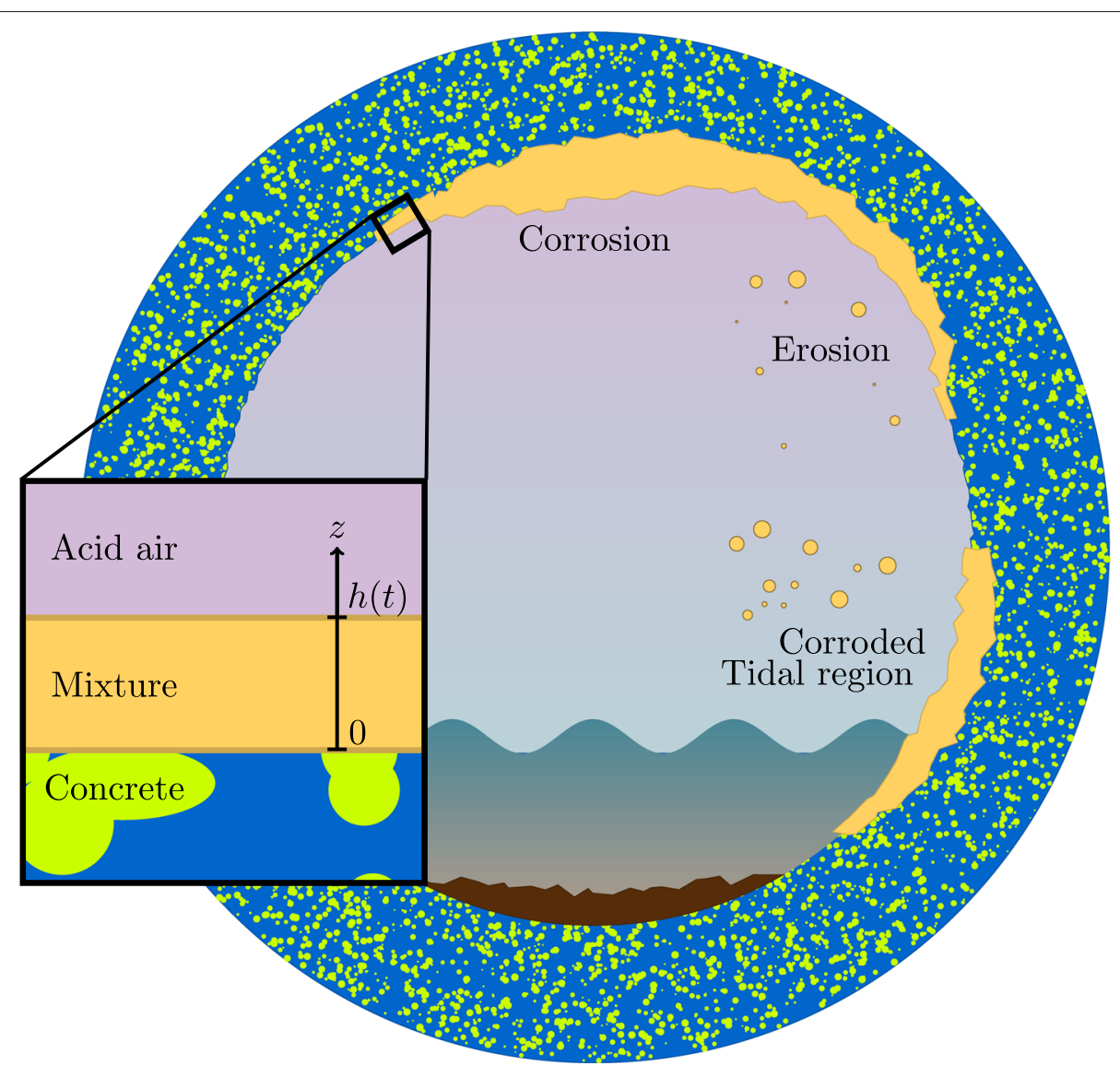

Fig. 1 A concrete sewer pipe is corroded by sulfuric acid containing air at the top and by the acidic sewage at the tidal region of the sewage-air interface. The sulfuric acid is created by biodegradation of bio-matter in sewage. Extended corrosion leads to erosion of the concrete and potentially to sewer pipe collapse. Our model is meant to describe the beginning of corrosion, as shown in the small square, allowing the simplifications from a pipe to a plate layer, as shown in the large square. This simplification reduces a 3-D concrete corrosion model into a 1-D model only dependent on the spatial variable $z$. The thickness, $h(t)$, of the mixture layer changes over time due to both influx of material and the chemical reaction in the mixture

of the constituent $\alpha$ with molecular mass $M_{\alpha}$. The mass $m_{\alpha}(g(t))$ of constituent $\alpha$ in $g(t)$ is given by

$$
m_{\alpha}(g(t))=M_{\alpha} n_{\alpha}(g(t))=\mathcal{M}_{\alpha} n_{\alpha}(g(t)) / N_{A},
$$

where $N_{A}$ denotes the Avogadro constant (i.e. $6.022 \times 10^{23}$ molecules per mole) and $\mathcal{M}_{\alpha}$ the molar mass of constituent $\alpha$. The total mass $m(g(t))$ of the mixture in $g(t)$ is given by

$$
m(g(t))=\sum_{\alpha} m_{\alpha}(g(t))
$$

A strictly positive integrable function $\rho_{\alpha}(\mathbf{x}, t)$, called the partial density of component $\alpha$, is defined by

$$
m_{\alpha}(g(t))=\int_{g(t)} \rho_{\alpha}(\mathbf{x}, t) \mathrm{d} V .
$$

The density of the mixture in the point $(\mathbf{x}, t)$ is

$$
\rho(\mathbf{x}, t)=\sum_{\alpha} \rho_{\alpha}(\mathbf{x}, t)
$$

Let $\tilde{\rho}_{\alpha}$ be the intrinsic density of component $\alpha$ (i.e. the density of the isolated component) and let $\phi_{\alpha}(\mathbf{x}, t)$ be its volume fraction, then

$$
\rho_{\alpha}(\mathbf{x}, t)=\tilde{\rho}_{\alpha} \phi_{\alpha}(\mathbf{x}, t), \quad \sum_{\alpha} \phi_{\alpha}(\mathbf{x}, t)=1 \text { for all }(\mathbf{x}, t) \in g(t) .
$$

We assume that the constituents of the mixture are incompressible. Hence, the intrinsic densities $\tilde{\rho}_{\alpha}$ are uniform constants.

\subsection{Balance laws}

Following [7] and in analogy with [6, 27], we describe the time evolution of our 3-component mixture by means of two sets of global balance laws for each component of the mixture: one for mass and one for momentum conservation. We assume that the chemical reaction is an isothermal process; the conservation of energy is then automatically satisfied. 
The conservation of the partial mass for component $\alpha$ is formulated as the balance law for the partial density $\rho_{\alpha}=\rho_{\alpha}(\mathbf{x}, t)$ in the form:

$$
\begin{aligned}
\frac{\mathrm{d}}{\mathrm{d} t} m_{\alpha}(g(t)) & =\frac{\mathrm{d}}{\mathrm{d} t} \int_{g(t)} \rho_{\alpha}(\mathbf{x}, t) \mathrm{d} V \\
& =\int_{\partial g(t)} \delta_{\alpha} \nabla \rho_{\alpha}(\mathbf{x}, t) \cdot \mathrm{d} S+\int_{g(t)} R_{\alpha}(\mathbf{x}, t) \mathrm{d} V .
\end{aligned}
$$

In this balance law, the outward flux is given by Fick's law of diffusion, and equals $-\delta_{\alpha} \nabla \rho_{\alpha}$, where $\delta_{\alpha}$ is the diffusion coefficient of the $\alpha$-th component. The production term by chemical reaction $R_{\alpha}$ acts either as a source (sink) when the constituent is being produced (consumed) in the chemical reaction. Remark, Eq. (6) indicates that $g(t)$ is NOT an element of material volume for a single constituent, but it is an element of material volume for the collection of all constituent particles. Hence, in Eq. (6), summing up over $\alpha$ and using that $g(t)$ is an element of material volume such that $m(g(t))$ is constant, we obtain

$$
\begin{aligned}
0= & \frac{\mathrm{d}}{\mathrm{d} t} \int_{g(t)} \rho(\mathbf{x}, t) \mathrm{d} \mathbf{x}=\int_{\partial g(t)} \sum_{\alpha}\left(\delta_{\alpha} \nabla \rho_{\alpha}\right)(\mathbf{x}, t) \cdot \mathrm{d} \mathbf{s} \\
& +\int_{g(t)} \sum_{\alpha} R_{\alpha}(\mathbf{x}, t) \mathrm{d} \mathbf{x} .
\end{aligned}
$$

Note, the elements $g(t)$ can overlap due to the diffusion term, but that it does not violate the material element rules.

Since a chemical reaction is inherently a massconserving process, we obtain $\sum_{\alpha} R_{\alpha}=0$. Thus this global mass conservation is satisfied if $\sum_{\alpha} \delta_{\alpha} \nabla \rho_{\alpha}=$ $\sum_{\alpha} \delta_{\alpha} \tilde{\rho}_{\alpha} \nabla \phi_{\alpha}=0$, a compatibility condition for the allowed types of internal diffusion processes. This is satisfied if, for instance, $\delta_{\alpha}=\delta / \tilde{\rho}_{\alpha}$. Hence, $\delta=0$ (no internal diffusion) would suffice.

Conservation of linear momentum for the component $\alpha$ is formulated as

$$
\begin{aligned}
\frac{\mathrm{d}}{\mathrm{d} t} \int_{g(t)}\left(\rho_{\alpha} \mathbf{v}_{\alpha}\right)(\mathbf{x}, t) \mathrm{d} V= & \int_{\partial g(t)} \mathbb{T}_{\alpha}(\mathbf{x}, t) \cdot \mathrm{d} S \\
& +\int_{g(t)} \mathbf{B}_{\alpha}(\mathbf{x}, t) \mathrm{d} V,
\end{aligned}
$$

where $\rho_{\alpha} \mathbf{v}_{\alpha}$ is the linear momentum density of the component $\alpha$, while the outward flux is given by the partial stress tensor $\mathbb{T}_{\alpha}$ and the production term by the internal linear momentum production $\mathbf{B}_{\alpha}$. The latter two terms will be specified in the next subsection. Since in our setting the mechanical processes and flow dynamics are slow, we assume a quasi-static regime. This implies that the inertia term on the left-hand side in Eq. (8) may be neglected. Moreover, the sum of the internal momentum-production terms $\mathbf{B}_{\alpha}$ must be zero, i.e. $\sum_{\alpha} \mathbf{B}_{\alpha}=0$, by Newton's third law.

\subsection{Local equations and jump conditions}

The global balance equations can in the usual way be converted into local balance equations and jump conditions across a singular surface $\Sigma(t)$. Thus, we obtain from Eq. (6) the local partial mass balance equations (or continuity equations):

$$
\frac{\partial \rho_{\alpha}}{\partial t}+\nabla \cdot\left(\rho_{\alpha} \mathbf{v}_{\alpha}\right)-\delta_{\alpha} \Delta \rho_{\alpha}=R_{\alpha}
$$

together with the jump condition at $\Sigma(t)$

$$
\llbracket \rho_{\alpha}\left(\mathbf{V} \cdot \mathbf{n}-\mathbf{v}_{\alpha} \cdot \mathbf{n}\right)+\delta_{\alpha} \nabla \rho_{\alpha} \cdot \mathbf{n} \rrbracket=0
$$

where [[.]] denotes the outward jump across the surface $\Sigma(t), \mathbf{V}$ the velocity of $\Sigma(t)$, and $\mathbf{n}$ the outward unit normal on $\Sigma(t)$. We rewrite the mass equations by elimination of $\rho_{\alpha}$ in favour of $\phi_{\alpha}$ yielding

$$
\frac{\partial \phi_{\alpha}}{\partial t}+\nabla \cdot\left(\phi_{\alpha} \mathbf{v}_{\alpha}\right)-\delta_{\alpha} \Delta \phi_{\alpha}=\frac{R_{\alpha}}{\tilde{\rho}_{\alpha}} .
$$

Summing Eq. (11) over all $\alpha$, we obtain

$$
\begin{aligned}
\sum_{\alpha} \nabla \cdot\left(\phi_{\alpha} \mathbf{v}_{\alpha}\right) & =\sum_{\alpha}\left(\delta_{\alpha} \Delta \phi_{\alpha}+\frac{R_{\alpha}}{\tilde{\rho}_{\alpha}}\right) \\
& =\sum_{\alpha} \frac{1}{\tilde{\rho}_{\alpha}}\left(\delta \Delta \phi_{\alpha}+R_{\alpha}\right),
\end{aligned}
$$

with use of $\delta_{\alpha}=\delta / \tilde{\rho}_{\alpha}$. We refer to Eq. (12) as the incompressibility condition. Later we shall use Eq. (12) to replace one of the three mass equations (e.g. for $\alpha=2$, and then use $\left.\phi_{2}=1-\phi_{1}-\phi_{3}\right)$.

Analogously, the quasi-static momentum balance yields

$$
\nabla \cdot \mathbb{T}_{\alpha}+\mathbf{B}_{\alpha}=0
$$

with the jump condition

$$
\llbracket\left[\mathbb{T}_{\alpha} \cdot \mathbf{n} \rrbracket=0 .\right.
$$

Summing Eq. (13) over all $\alpha$ and using $\mathbb{T}=\sum_{\alpha} \mathbb{T}_{\alpha}$, the total stress tensor, and $\sum_{\alpha} \mathbf{B}_{\alpha}=0$, we find

$$
\nabla \cdot \mathbb{T}=0
$$

Before we can evaluate the local momentum equations any further we have to make constitutive assumptions concerning the structure of $\mathbb{T}_{\alpha}$ and $\mathbf{B}_{\alpha}$.

The two solid components, $\alpha=(1,2)$ are modeled as linearly (visco)elastic media, the stress tensor $\mathbb{T}_{\alpha}$ of which is given by

$$
\mathbb{T}_{\alpha}=-\phi_{\alpha} p \mathbb{I}+\mathbb{T}_{\alpha}^{\mathrm{el}}+\mathbb{T}_{\alpha}^{\mathrm{ve}}
$$

where $p$ is the pressure (this pressure term is needed to compensate for the incompressibility assumption), II the 
unit tensor, $\mathbb{T}_{\alpha}^{\mathrm{el}}$ is the linear elastic part and $\mathbb{T}_{\alpha}^{\mathrm{ve}}$ the linear viscoelastic part. The first one is given by Hooke's law as

$$
\mathbb{T}_{\alpha}^{\mathrm{el}}=\lambda_{\alpha} \operatorname{Tr}\left(\mathbb{E}_{\alpha}\right) \mathbb{I}+2 \mu_{\alpha} \mathbb{E}_{\alpha} \quad \text { for } \alpha \in\{1,2\},
$$

where $\mathbb{E}_{\alpha}=\left(\nabla \mathbf{u}_{\alpha}+\left(\nabla \mathbf{u}_{\alpha}\right)^{\top}\right) / 2$ is the linear deformation tensor written in terms of the displacement $\mathbf{u}_{\alpha}, \operatorname{Tr}(A)$ means the trace of the matrix $A$, and $\lambda_{\alpha}$ and $\mu_{\alpha}$ are the corresponding Lamé parameters. The viscoelastic part is modeled such that Eq. (16) follows the Kelvin-Voigt model, see $[8,25]$, and has the general structure

$$
\mathbb{T}_{\alpha}^{\mathrm{ve}}=\sum_{\beta=1}^{2} \gamma_{\alpha \beta} \mathbb{D}_{\beta} \quad \text { for } \alpha \in\{1,2\},
$$

where $\mathbb{D}_{\alpha}=\left(\nabla \mathbf{v}_{\alpha}+\left(\nabla \mathbf{v}_{\alpha}\right)^{\top}\right) / 2$ is the rate of deformation tensor based on the velocity $\mathbf{v}_{\alpha}=\partial \mathbf{u}_{\alpha} / \partial t$, while the coefficients $\gamma_{\alpha \beta}$ are material constants that will be further specified below.

The internal linear momentum production represents the Stokes Drag ([27], Eq. (92)), i.e.

$$
\mathbf{B}_{\alpha}^{(S D)}=-\chi_{\alpha}\left(\mathbf{v}_{\alpha}-\mathbf{v}_{3}\right) \quad \text { for } \quad \alpha \in\{1,2\},
$$

and

$$
\mathbf{B}_{3}^{(S D)}=\sum_{\beta=1}^{2} \chi_{\beta}\left(\mathbf{v}_{\beta}-\mathbf{v}_{3}\right),
$$

such that $\sum_{\alpha} \mathbf{B}_{\alpha}^{(S D)}=0$. For an explicit definition of the material parameter $\chi_{\alpha}$, we refer to the note ${ }^{\dagger}$ in Table 1.

The fluid is modeled as an inviscid Newtonian fluid, possibly modified by an extra linear viscoelastic term, which in general is zero, except for the first of the four systems to be introduced next, i.e.

$$
\mathbb{T}_{3}=-\phi_{3} p \mathbb{I}+\mathbb{T}_{3}^{\mathrm{ve}} \text {. }
$$

The specification of $\gamma_{\alpha \beta}$ entering the structure of $\mathbb{T}_{\alpha}^{\mathrm{ve}}$ (cf. Eq. 18) differs for the four systems we introduce now:

1 System A: This system corresponds best to the evolution systems studied in [40], where conditions for the existence of weak solutions were obtained. Here, the individual constituents are assumed to be viscoelastic, such that the mixture as a whole remains purely elastic. For this, we choose $\gamma_{\alpha \beta}=\gamma_{\alpha}$ if $\beta=\alpha \in\{1,2\}$, and $\gamma_{\alpha \beta}=0$ if $\beta \neq \alpha$, resulting in

$$
\mathbb{T}_{\alpha}^{\mathrm{ve}}=\gamma_{\alpha} \mathbb{D}_{\alpha} \quad \text { for } \alpha \in\{1,2\} \text {. }
$$

Moreover, we take $\mathbb{T}_{3}^{\mathrm{ve}}$ such that

$$
\mathbb{T}_{3}^{\mathrm{ve}}=-\sum_{\alpha=1}^{2} \gamma_{\alpha} \mathbb{D}_{\alpha}=-\sum_{\alpha=1}^{2} \mathbb{T}_{\alpha}^{\mathrm{ve}},
$$

providing that $\mathbb{T}=\sum_{\alpha=1}^{3} \mathbb{T}_{\alpha}^{\mathrm{ve}}=0$.

2 System B: Here, $\gamma_{\alpha \beta}=0$ : the solid components are thus purely elastic and the fluid inviscid.
3 System C: As in System A, the solid components are intrinsic viscoelastic, but the fluid is inviscid, so $\mathbb{T}_{3}=-\phi_{3} p \mathbb{I}$, implying that the mixture as a whole is also viscoelastic. This has consequences on the pressure term $p$, as can be seen in the 1-D problem described in Section 3; see (41).

4 System D: In this case, we assume that the viscoelastic terms in the stresses are proportional to the differences in shear rates of the two solids so that these stresses are zero if the velocities, or displacements, of the solids are equal. Moreover, we let the sum of the two stresses equal zero and keep the fluid inviscid. Thus, the total stress is purely elastic. This results in the following choice for $\gamma_{\alpha \beta}$

$$
\gamma_{11}=\gamma_{22}=\gamma \text {, and } \gamma_{12}=\gamma_{21}=-\gamma .
$$

System A is well-posed mathematically (cf. [40]), but is possibly physically incorrect as the sulfuric acid viscoelastic stress is defined by the viscoelastic stress of the other components, see (23). System B is physically nice, but mathematically it needs an additional viscoelastic term to ensure the existence of weak solutions and FEM approximations. System $C$ combines the strong points of systems A and B. It is physically justified and mathematically sound. However, the mixture is viscoelastic, which is a behavior one would expect on unnaturally large timescales. System D is both mathematically and physically sound, supporting an elastic mixture, which favors timescales compatible with measurements.

The physical derivation of systems A, B, C and D indicate that only system $\mathrm{D}$ has the right physical properties at the desired timescales. Hence, from here on we will focus on system D from both analytical and numerical perspectives, for example when we judge solutions to exhibit realistic behaviors. To reduce complexity, we investigate a special situation leading effectively to a $1-\mathrm{D}$ version of system D.

\subsection{Chemical corrosion of concrete with sulphates}

The concrete corrosion we discuss here refers to sulfuric acid reacting with slaked lime to create gypsum. The reaction mechanism is very complex, leading to ettringite growth, e.g. see [37]. In this paper, the chemical reaction mechanism takes the simplified form (s: solid, f: fluid)

$$
\begin{gathered}
\text { slaked lime (s) } \\
\mathrm{Ca}(\mathrm{OH})_{2}
\end{gathered}+\begin{gathered}
\text { sulfuric acid (f) } \\
\mathrm{H}_{2} \mathrm{SO}_{4}
\end{gathered} \rightarrow \underset{\mathrm{CaSO}_{4} \cdot 2 \mathrm{H}_{2} \mathrm{O} .}{\operatorname{gypsum}(\mathrm{s})}
$$

Hence, the stoichiometric coefficients $N_{\alpha}$ are $N_{1}=1$ and $N_{2}=N_{3}=-1$.

The chemical reaction, as shown above, is the net reaction and does not reflect the full complexity of all the intermediate steps necessary for this reaction. The complexity is encompassed in a single rate equation. A similar reaction as above but with calcite, $\mathrm{CaCO}_{3}$, instead of 
Table 1 Table with numerical values of material constants, normalization constants, dimensionless parameters, and numerical parameters

\begin{tabular}{|c|c|c|c|c|c|c|}
\hline & \multicolumn{3}{|c|}{ Material constants } & & \multicolumn{2}{|c|}{ Dimensionless parameters } \\
\hline & Value & (MKS unit) & Reference & & Value & Definition \\
\hline$E_{1}$ & $1.60 \cdot 10^{9}$ & $\left(\mathrm{~kg} / \mathrm{m} \mathrm{s}^{2}\right)$ & [32] & $E_{1}$ & 0.038 & $E_{1} / E$ \\
\hline$E_{2}$ & $4.20 \cdot 10^{10}$ & $\left(\mathrm{~kg} / \mathrm{ms}^{2}\right)$ & [41] & $E_{2}$ & 1.00 & $E_{2} / E$ \\
\hline$\chi_{1}$ & $2.67 \cdot 10^{10}$ & $\left(\mathrm{~kg} / \mathrm{m}^{3} \mathrm{~s}\right)$ & $\dagger$ & $\chi_{1}$ & 1.00 & $\chi_{1} / \chi$ \\
\hline$\chi_{2}$ & $2.67 \cdot 10^{10}$ & $\left(\mathrm{~kg} / \mathrm{m}^{3} \mathrm{~s}\right)$ & $\dagger$ & $x_{2}$ & 1.00 & $x_{2} / x$ \\
\hline $\mathcal{J}_{2}$ & $0.326 \cdot 10^{-5}$ & $(\mathrm{~m} / \mathrm{s})$ & * & $\mathcal{J}_{2}$ & 0.40 & $\mathcal{J}_{2} / J$ \\
\hline $\mathcal{J}_{3}$ & $1.632 \cdot 10^{-5}$ & $(\mathrm{~m} / \mathrm{s})$ & * & $\mathcal{J}_{3}$ & 2.00 & $\mathcal{J}_{3} / J$ \\
\hline$\gamma_{1}$ & $3.604 \cdot 10^{10}$ & $(\mathrm{~kg} / \mathrm{ms})$ & * & $\gamma_{1}$ & 0.50 & $\gamma_{1} / \gamma$ \\
\hline$\gamma_{2}$ & $3.604 \cdot 10^{10}$ & $(\mathrm{~kg} / \mathrm{ms})$ & * & $\gamma_{2}$ & 0.50 & $\gamma_{2} / \gamma$ \\
\hline$A_{1}$ & $0.821 \cdot 10^{-3}$ & $(1 / \mathrm{m})$ & * & $A_{1}$ & 0.50 & $A_{1} / A$ \\
\hline$A_{2}$ & $0.821 \cdot 10^{-3}$ & $(1 / m)$ & * & $A_{2}$ & 0.50 & $A_{2} / A$ \\
\hline$\tilde{\rho}_{1}$ & $2.32 \cdot 10^{3}$ & $\left(\mathrm{~kg} / \mathrm{m}^{3}\right)$ & [20] & $\phi_{1 s a t}$ & 1.00 & \\
\hline$\tilde{\rho}_{2}$ & $2.21 \cdot 10^{3}$ & $\left(\mathrm{~kg} / \mathrm{m}^{3}\right)$ & {$[20]$} & $\phi_{3 \text { thr }}$ & 0.00 & \\
\hline$\tilde{\rho}_{3}$ & $1.84 \cdot 10^{3}$ & $\left(\mathrm{~kg} / \mathrm{m}^{3}\right)$ & [20] & $\phi_{2 \text { res }}$ & 1.00 & \\
\hline $\mathcal{M}_{1}$ & 0.172164 & $(\mathrm{~kg} / \mathrm{mol})$ & {$[20]$} & $\phi_{3 \text { res }}$ & 1.00 & \\
\hline $\mathcal{M}_{2}$ & 0.074093 & $(\mathrm{~kg} / \mathrm{mol})$ & [20] & $\kappa_{1}$ & 23.00 & Eq. (51) \\
\hline $\mathcal{M}_{3}$ & 0.098079 & $(\mathrm{~kg} / \mathrm{mol})$ & {$[20]$} & $\kappa_{3}$ & 13.50 & Eq. (51) \\
\hline$\delta$ & 5.10 & (kg/m s) & * & $\delta_{1}$ & 1.00 & $\delta_{1} / K H^{2}$ \\
\hline$\delta_{1}$ & $2.20 \cdot 10^{-3}$ & $\left(\mathrm{~m}^{2} / \mathrm{s}\right)$ & 末 & $\delta_{2}$ & 1.05 & $\delta_{2} / K H^{2}$ \\
\hline$\delta_{2}$ & $2.31 \cdot 10^{-3}$ & $\left(\mathrm{~m}^{2} / \mathrm{s}\right)$ & $\ddagger$ & $\delta_{3}$ & 1.26 & $\delta_{3} / K H^{2}$ \\
\hline$\delta_{3}$ & $2.77 \cdot 10^{-3}$ & $\left(\mathrm{~m}^{2} / \mathrm{s}\right)$ & $\ddagger$ & & & \\
\hline \multirow[t]{3}{*}{ k } & $1.00 \cdot 10^{-6}$ & $\left(\mathrm{~m}^{3} / \mathrm{mol} \mathrm{s}\right)$ & [3] & & & \\
\hline & \multicolumn{3}{|c|}{ Normalization Constants } & & \multicolumn{2}{|c|}{ Numerical Parameters } \\
\hline & Value & (MKS unit) & Definition & & Value & Definition \\
\hline H & $1.643 \cdot 10^{0}$ & (m) & $h(0)$ & $\Delta t$ & 0.001 & \\
\hline K & $0.816 \cdot 10^{-3}$ & $(1 / s)$ & Eq. (44) & $t_{f}$ & 0.5 & $T_{f} / T$ \\
\hline$S_{K}$ & -1 & $(-)$ & Eq. (44) & $1 / \Delta z$ & 300 & \\
\hline$\chi$ & $2.67 \cdot 10^{10}$ & $\left(\mathrm{~kg} / \mathrm{m}^{3} \mathrm{~s}\right)$ & $\chi_{1}$ & $\phi_{\min }$ & $10^{-5}$ & \\
\hline$E$ & $4.20 \cdot 10^{10}$ & $\left(\mathrm{~kg} / \mathrm{m} \mathrm{s}^{2}\right)$ & $E_{2}$ & $V_{\max }$ & $10^{6}$ & \\
\hline$T$ & 1.716 & (s) & $\chi H^{2} / E$ & & & \\
\hline U & $2.300 \cdot 10^{-3}$ & (m) & $\chi H^{3} K / E$ & & & \\
\hline V & $1.341 \cdot 10^{-3}$ & $(\mathrm{~m} / \mathrm{s})$ & $H K$ & & & \\
\hline J & $0.816 \cdot 10^{-3}$ & $(\mathrm{~m} / \mathrm{s})$ & $H K$ & & & \\
\hline$\gamma$ & $7.208 \cdot 10^{10}$ & $(\mathrm{~kg} / \mathrm{m} \mathrm{s})$ & $x H^{2}$ & & & \\
\hline$\epsilon$ & 0.0014 & $(-)$ & $\chi H^{2} K / E$ & & & \\
\hline
\end{tabular}

* An experimental value of this parameter is unknown to us; we have chosen their values such that their dimensionless values are of order one of magnitude. Specifically, the values of $J_{2}$ and $J_{3}$ are so large that they guarantee growth of the layer; see also remark just below Eq. (55)

$\dagger$ We estimated the values of $\chi_{\alpha}$ from the Darcy law with $\chi_{\alpha}=\mu / k_{0}$ with $\mu$ the dynamic viscosity of sulfuric acid (value of $26.7 \cdot 10^{-3} \mathrm{~kg} / \mathrm{ms}$, see ([15], p. 304-305) and $k_{0}$ the average pore size or permeability (about $1 \mu \mathrm{m}^{2}=10^{-12} \mathrm{~m}^{2}$ ); see $[11,24]$

$¥$ We used $\delta_{\alpha}=\delta / \tilde{\rho}_{\alpha}$ for $\alpha=1,2,3$

slaked lime has been treated in [5]. Therefore, we assume a rate equation similar to the one in [5], i.e.

$r=k \mathcal{F}=k \mathcal{L}\left(\left[\mathrm{H}_{2} \mathrm{SO}_{4}\right]-C_{e q}\right) \mathcal{L}\left(C_{\max }-[\right.$ gypsum $\left.]\right)$, where we denote $\mathcal{L}(u)=u \mathcal{H}(u)$ with $\mathcal{H}$ the Heaviside function, $k$ is the volumetric reaction rate (in $\left[\mathrm{m}^{3} / \mathrm{mol} \cdot \mathrm{s}\right]$ ), $[f]$ the molar concentration of $f, C_{e q}$ the dissolution equilibrium molar concentration of the sulfuric acid, and 
$C_{\max }$ the maximum precipitation molar concentration of gypsum.

The mass production term $R_{\alpha}=R_{\alpha}(\mathbf{x}, t)$ is given by

$$
R_{\alpha}(\mathbf{x}, t)=N_{\alpha} \mathcal{M}_{\alpha} r(\mathbf{x}, t) \quad \text { for }(\mathbf{x}, t) \in g(t),
$$

which satisfies $\sum_{\alpha} R_{\alpha}=0$. Moreover, Eq. (26) implies that the volume fraction production can be written as

$$
\frac{R_{\alpha}(\mathbf{x}, t)}{\tilde{\rho}_{\alpha}}=\frac{\mathcal{M}_{\alpha} N_{\alpha}}{\tilde{\rho}_{\alpha}} \tilde{k} \frac{\tilde{\rho}_{1} \tilde{\rho}_{3}}{\mathcal{M}_{1} \mathcal{M}_{3}} \mathcal{F}(\mathbf{x}, t) \quad \text { for }(\mathbf{x}, t) \in g(t),
$$

with

$$
\mathcal{F}=\mathcal{L}\left(\phi_{3}-\phi_{3, \text { thr }}\right) \mathcal{L}\left(\phi_{1, \text { sat }}-\phi_{1}\right),
$$

where $\phi_{1, \text { sat }}$ is the gypsum saturation level, while $\phi_{3, t h r}$ represents the sulfuric acid dissolution threshold.

\subsection{Initial and boundary conditions}

We consider a mixture body, placed freely in space, and initially in a homogeneous, undeformed state, free of stress and movement. This yields the initial conditions:

$\phi_{\alpha}(\mathbf{x}, 0)=\phi_{\alpha 0}(\mathbf{x}) \quad$ and $\quad \mathbf{u}_{\alpha}(\mathbf{x}, 0)=0 \quad$ for $(\mathbf{x}, 0) \in g(0)$,

where $\phi_{\alpha 0}=\rho_{\alpha 0} / \tilde{\rho}_{\alpha}$ are prescribed initial concentration values.

We wish to point out here that, although $\mathbf{u}_{3}\left(\mathbf{x}, 0^{+}\right)=0$, there is a jump in the velocity $\mathbf{v}_{3}$, which is inherent to the quasi-static approximation we used.

Due to the influx of material (acid and/or gypsum) across the boundary and the chemical reactions, the domain $G$ will change as time elapses, i.e. $G=\mathcal{G}(t)$ as does its boundary $\partial G=\partial \mathcal{G}(t)$. However, in consistency with the linear deformation assumption, the boundary condition may be considered to hold on the undeformed (reference) boundary. The space outside the domain can contain any of the constituents with a concentration $\phi_{\alpha}^{+}$. The influx is assumed to be proportional to the concentration difference $\llbracket \phi_{\alpha} \rrbracket$ across $\partial G$, provided this difference is positive. The boundary is semi-permeable for all constituents $\alpha$, allowing only one-sided transfer from outside the domain into the domain if $\phi_{\alpha}^{+}>\left.\phi_{\alpha}\right|_{\partial G}$. This leads to the boundary condition (compare with Eq. (10)), holding for $t>0$

$$
\phi_{\alpha}\left(\mathbf{v}_{\alpha}-\mathbf{V}\right) \cdot \mathbf{n}+\delta_{\alpha} \nabla \phi_{\alpha} \cdot \mathbf{n}=\mathcal{J}_{\alpha}\left(\mathcal{L}\left(\llbracket \phi_{\alpha} \rrbracket\right)\right) \quad \text { at } \partial G,
$$

where $\mathbf{n}$ denotes the outward normal on $\partial G, \mathbf{V}$ the velocity of the boundary, $\llbracket \phi_{\alpha} \rrbracket=\phi_{\alpha}^{+}-\phi_{\alpha}$ with $\phi_{\alpha}^{+}$the volume fraction of $\alpha$ outside $\partial G$ and $\phi_{\alpha}$ just inside it, while $\mathcal{J}_{\alpha}$ is a material constant. If, on the other hand, for certain $\alpha$, we have $\phi_{\alpha}^{+}<\left.\phi_{\alpha}\right|_{\partial G}$, then the influx is zero (due to the semi-permeability), leading to the boundary condition ${ }^{1}$

$$
\nabla \phi_{\alpha} \cdot \mathbf{n}=0 \quad \text { at } \partial G
$$

If the outer space contains only one constituent, say $\beta$, then Eq. (31) holds for the two values $\alpha \neq \beta$, but then $\sum_{\alpha} \phi_{\alpha}=1$ yields directly that also $\nabla \phi_{\beta} \cdot \mathbf{n}=0$, and thus the second term on the left-hand side of Eq. (30) vanishes, so that this boundary condition for $\alpha \rightarrow \beta$ becomes

$$
\phi_{\beta}\left(\mathbf{v}_{\beta}-\mathbf{V}\right) \cdot \mathbf{n}=\mathcal{J}_{\beta} \mathcal{L}\left(\llbracket \phi_{\beta} \rrbracket\right) \quad \text { at } \partial G,
$$

the right-hand side of which is greater than zero if $\phi_{\beta}^{+}>\phi_{\beta}$.

For the free unloaded body that we will consider in this paper, the boundary is free of stress, which implies

$$
\mathbb{T} \cdot \mathbf{n}=\sum_{\alpha} \mathbb{T}_{\alpha} \cdot \mathbf{n}=0 \quad \text { at } \partial G .
$$

If, for some $\alpha$, we have $\phi_{\alpha}^{+}<\phi_{\alpha}$, then the flux is zero and hence, the boundary condition (6) reduces to

$$
\mathbf{v}_{\alpha} \cdot \mathbf{n}=\mathbf{V} \cdot \mathbf{n} \text { at } \partial G .
$$

However, instead of (30) a different boundary condition, particular for the solid constituents $(\alpha=1,2)$ is used, namely

$$
\left(\nabla_{\mathbf{x}} \mathbf{u}_{\alpha} \cdot \mathbf{n}\right)^{\top} \cdot \mathbf{n}=A_{\alpha}\left(\mathbf{u}_{\alpha}-\mathbf{W}\right) \cdot \mathbf{n} \quad \text { at } \partial G .
$$

In Eq. (35), $\mathbf{W}$ denotes the displacement vector of the boundary such that $\mathbf{V}(t)=\mathrm{d} \mathbf{W} / \mathrm{d} t$. In [40] it was shown that a finite positive value of $A_{\alpha}$ is useful to prove existence of a realistic numerical approximation of weak solutions. Note that in the limit $A_{\alpha} \rightarrow \infty$ the boundary condition $\mathbf{u}_{\alpha}=\mathbf{W}$ is retrieved. On the other hand, in the opposite limit $A_{\alpha} \rightarrow 0$ the boundary condition becomes the homogeneous Neumann boundary condition

$$
\left(\nabla_{\mathbf{x}} \mathbf{u}_{\alpha} \cdot \mathbf{n}\right)^{\top} \cdot \mathbf{n}=0,
$$

which is equivalent to requiring that the partial normal stress of constituent $\alpha$ is zero.

\subsection{Summary of the model equations}

Based on the discussion from the preceding sections, we are now able to formulate complete 3-D systems of equations and boundary conditions for the reacting, diffusing and deforming 3-component continuum mixture. From the four systems presented before, we opt for System $\mathrm{D}$. The internal unknowns (6 in number, of which 3 scalar and 3 vectorial) are $\left\{\phi_{1}, \phi_{3}, \mathbf{u}_{1}, \mathbf{u}_{2}, \mathbf{v}_{3}, p\right\}$, with $\phi_{2}=1-\phi_{1}-\phi_{3}$, for which we have a set of balance equations, following from successively the local mass balances, the incompressibility condition and the 3 local momentum balances. Together with the constitutive 
equations for $\mathbb{T}_{\alpha}$ and $\mathbf{B}_{\alpha}$, given by Eqs. (16) to (21) and (24), we obtain for $t>0$ and $\mathbf{x} \in G$ :

$$
\begin{aligned}
\frac{\partial \phi_{\alpha}}{\partial t}+\nabla \cdot\left(\phi_{\alpha} \mathbf{v}_{\alpha}\right)-\delta_{\alpha} \Delta \phi_{\alpha} & =\frac{R_{\alpha}}{\tilde{\rho}_{\alpha}} \quad \text { for } \alpha \in\{1,2,3\}, \\
\nabla \cdot\left(\sum_{\alpha=1}^{3} \phi_{\alpha} \mathbf{v}_{\alpha}\right)-\sum_{\alpha=1}^{3} \delta_{\alpha} \Delta \phi_{\alpha} & =\sum_{\alpha=1}^{3} \frac{R_{\alpha}}{\tilde{\rho}_{\alpha}}, \\
\nabla\left(-\phi_{\alpha} p+\left[\lambda_{\alpha}+\mu_{\alpha}\right] \nabla \cdot \mathbf{u}_{\alpha}\right)+\mu_{\alpha} \Delta \mathbf{u}_{\alpha} & =\chi_{\alpha}\left(\mathbf{v}_{\alpha}-\mathbf{v}_{3}\right)-\sum_{\beta=1}^{3} \gamma_{\alpha \beta} \Delta \mathbf{v}_{\beta}, \alpha \neq 3, \\
\nabla\left(-\phi_{3} p\right) & =-\sum_{\beta=1}^{2}\left[\chi_{\beta}\left(\mathbf{v}_{\beta}-\mathbf{v}_{3}\right)+\gamma_{3 \beta} \Delta \mathbf{v}_{\beta}\right],
\end{aligned}
$$

where $\mathbf{v}_{\beta}=\partial_{t} \mathbf{u}_{\beta}=\partial \mathbf{u}_{\beta} / \partial t$ for $\beta \in 1$, 2. Combining the three momentum equations, and using that $\sum_{\alpha=1}^{3} \phi_{\alpha}=1$, we obtain the global momentum equation:

$$
\begin{aligned}
\nabla\left(-p+\sum_{\alpha=1}^{2}\left(\lambda_{\alpha}+\mu_{\alpha}\right) \nabla \cdot \mathbf{u}_{\alpha}\right) & +\sum_{\alpha=1}^{2} \mu_{\alpha} \Delta \mathbf{u}_{\alpha} \\
& +\sum_{\alpha=1}^{3} \sum_{\beta=1}^{3} \gamma_{\alpha \beta} \Delta \mathbf{v}_{\beta}=0,
\end{aligned}
$$

in which the $\gamma$-term is only non-zero for System C.

We can replace Eq. (37d) describing the fluid motion by this global equation, and then determine the pressure $p$ from it with the aid of the stress boundary condition.

The initial conditions are given in Eq. (29) and the necessary boundary conditions are Eqs. (30), (31), (33) and (35).

\section{Dimension reduction: 1-D model of a concrete plate-layer}

We reduce the 3-D model of Section 2 to a simpler 1D problem, namely a flat plate-layer of concrete of initial thickness $H$, which is exposed at its upper side to acidic air due to the presence of droplets of sulfuric acid. The bottom of the plate layer is fixed on a rigid ground space of non-reacting concrete having a fixed concentration of lime. The material of the layer (concrete) is a mixture of gypsum $(\alpha=1)$, lime $(\alpha=2)$ and sulfuric acid $(\alpha=3)$. Initially, i.e. for $t<0$, the layer is in a homogeneous, undeformed, and stress-free state, where the sulfuric acid has penetrated the concrete and has already partially reacted to create gypsum, such that $\phi_{\alpha 0}>0$ for $\alpha=(1,2,3)$. The external space both below and above the plate is free of stress. As the layer is created in a homogeneous and uniform way, and the acid is in equilibrium, we can forget about the tangential directions and only focus on the normal $(z)$ direction. Hence, a 1-D plate-layer model is sufficient to model a 3-D sewer pipe as already explained in the Introduction.
From $t>0$ onwards, the inflow of lime from below and acid from above into the plate takes place and chemical reactions start; here it is assumed that the concentrations $\phi_{2}^{-}$, of lime in the ground space, and $\phi_{3}^{+}$, of acid in the air above the plate, are greater than $\phi_{20}$ and $\phi_{30}$, respectively, resulting in an inflow of lime and acid. Due to the combination of inflow and the chemical reactions, the plate grows, as is experimentally observed in $[19,38]$, and the thickness of the plate increases to a value $h(t)>H=h(0)$ at time $t>0$. We consider only a time span from $t=0$ to a final time $t_{f}$ in which the growth remains small, i.e. such that $(h(t)-H) / H \ll 1$. Thus justifying our linear deformation assumption. A direct consequence of this assumption is that we may apply the boundary conditions at $z=H$ instead of at $z=h(t)$. All field variables are only dependent on $z$ and $t$, and the only displacement components are $u_{\alpha}=u_{\alpha}(z, t)=\mathbf{u}_{\alpha} \cdot \mathbf{e}_{z}$, with $\mathbf{e}_{z}$ the unit vector in the $z$-direction. This leads us to our 1-D model, valid for all four systems. Before recapitulating the resulting set of equations, we first use the global equation of equilibrium for the total stress Eq. (38), which in 1-D version reads

$$
\partial_{z}\left(-p+E_{1} \partial_{z} u_{1}+E_{2} \partial_{z} u_{2}\right)=0
$$

where $E_{1(2)}=\lambda_{1(2)}+2 \mu_{1(2)}$ is the Young's modulus of the solid constituent. Since the upper plane $z=H$ is free of stress, we have, for the moment for Systems A, B, and D only,

$$
\left(-p+E_{1} \partial_{z} u_{1}+E_{2} \partial_{z} u_{2}\right)(H, t)=0
$$

which, in combination with the equation above, implies that the total stress must be zero everywhere in the plate, yielding

$p(z, t)=E_{1} \partial_{z} u_{1}(z, t)+E_{2} \partial_{z} u_{2}(z, t)$ for $z \in[0, H]$ and $t \geq 0$.

This result holds for Systems A, B, and D, but for System $\mathrm{C}$ an extended expression is found, because in System C the total stress contains a viscoelastic part. Due to this, we get here

$$
\begin{gathered}
p=E_{1} \partial_{z} u_{1}+E_{2} \partial_{z} u_{2}+\gamma_{1} \partial_{z} \partial_{t} u_{1}+\gamma_{2} \partial_{z} \partial_{t} u_{2} \\
\quad \text { for } z \in[0, H] \quad \text { and } t \geq 0
\end{gathered}
$$

which further on leads to the expressions $\tilde{\gamma}_{\alpha \beta}$; see Eq. (45). After the elimination of $p$ from Eqs. (37c) and (37d), the set of unknown variables in the one-dimensional model is 


\section{$\left\{\phi_{1}, \phi_{3}, u_{1}, u_{2}, v_{3}\right\}(z, t)$,}

for $z \in(0, H)$ and $t \in\left(0, t_{f}\right)$. Reducing Eqs. (37a) to (37d) to their 1-D version, eliminating $p$, and inserting the volume fraction production $R_{\alpha}$ due to chemical reactions given by Eq. (27), we obtain the following 1-D model:

$$
\begin{aligned}
& \partial_{t} \phi_{1}+\partial_{z}\left(\phi_{1} \partial_{t} u_{1}\right)-\delta_{1} \partial_{z}^{2} \phi_{1}=\frac{N_{1} \tilde{\rho}_{3}}{\mathcal{M}_{3}} k \mathcal{F}\left(\phi_{1}, \phi_{3}\right), \\
& \partial_{t} \phi_{3}+\partial_{z}\left(\phi_{3} \nu_{3}\right)-\delta_{3} \partial_{z}^{2} \phi_{3}=\frac{N_{3} \tilde{\rho}_{1}}{\mathcal{M}_{1}} k \mathcal{F}\left(\phi_{1}, \phi_{3}\right), \\
& \partial_{z}\left(\phi_{1} \partial_{t} u_{1}+\phi_{2} \partial_{t} u_{2}+\phi_{3} \nu_{3}\right)-\sum_{\alpha=1}^{3} \delta_{\alpha} \partial_{z}^{2} \phi_{\alpha}=S_{K} K \mathcal{F}\left(\phi_{1}, \phi_{3}\right), \\
& \partial_{t} u_{1}-\frac{E_{1}}{\chi_{1}} \partial_{z}^{2} u_{1}-\frac{\gamma_{11}}{\chi_{1}} \partial_{z}^{2} \partial_{t} u_{1}-\frac{\gamma_{12}}{\chi_{1}} \partial_{z}^{2} \partial_{t} u_{2}=\nu_{3}-\partial_{z}\left(\phi_{1} \frac{E_{1}}{\chi_{1}} \partial_{z} u_{1}+\phi_{1} \frac{E_{2}}{\chi_{1}} \partial_{z} u_{2}\right), \\
& \partial_{t} u_{2}-\frac{E_{2}}{\chi_{2}} \partial_{z}^{2} u_{2}-\frac{\gamma_{22}}{\chi_{2}} \partial_{z}^{2} \partial_{t} u_{2}-\frac{\gamma_{21}}{\chi_{2}} \partial_{z}^{2} \partial_{t} u_{1}=v_{3}-\partial_{z}\left(\phi_{2} \frac{E_{1}}{\chi_{2}} \partial_{z} u_{1}+\phi_{2} \frac{E_{2}}{\chi_{2}} \partial_{z} u_{2}\right),
\end{aligned}
$$

where $\mathcal{F}\left(\phi_{1}, \phi_{3}\right)$ is given in Eq. (28), $\delta_{\alpha}=\delta / \tilde{\rho}_{\alpha}$, $\phi_{2}=1-\phi_{1}-\phi_{3}$, and

$K=\left(\sum_{\alpha=1}^{3} \frac{N_{\alpha} \mathcal{M}_{\alpha}}{\tilde{\rho}_{\alpha}}\right) \frac{\tilde{\rho}_{1} \tilde{\rho}_{3}}{\mathcal{M}_{1} \mathcal{M}_{3}} k, \quad S_{K}=\operatorname{sgn}\left(\sum_{\alpha=1}^{3} \frac{N_{\alpha} \mathcal{M}_{\alpha}}{\tilde{\rho}_{\alpha}}\right)$.

Moreover, $\gamma_{11}=\gamma_{1}, \gamma_{22}=\gamma_{2}, \gamma_{12}=\gamma_{21}=0$ for System A, $\gamma_{11}=\gamma_{22}=\gamma_{12}=\gamma_{21}=0$ for System B, and $\gamma_{11}=\gamma_{22}=-\gamma_{12}=-\gamma_{21}=\gamma$ for System D. For System $\mathrm{C}$ one has, due to the additional terms in $p$, the effective coefficients $\tilde{\gamma}$ defined by

$$
\begin{aligned}
& \tilde{\gamma}_{11}=\left(1-\phi_{1}\right) \gamma_{1}, \tilde{\gamma}_{12}=-\phi_{1} \gamma_{2}, \\
& \tilde{\gamma}_{21}=-\phi_{2} \gamma_{1}, \quad \tilde{\gamma}_{22}=\left(1-\phi_{2}\right) \gamma_{2},
\end{aligned}
$$

instead of $\gamma$. Since these effective coefficients depend on the volume fractions $\phi_{1,2}$ the (numerical) analysis of this system becomes more complicated than for the other systems.

The initial conditions at $t=0$ are

$$
\phi_{1}=\phi_{10}, \quad \phi_{3}=\phi_{30}, \quad u_{1}=u_{2}=0 .
$$

As boundary conditions we have for $t>0$ at $z=0$ :

$$
\partial_{z} \phi_{1}=\partial_{z} \phi_{3}=u_{1}=v_{3}=0, \quad \phi_{2} \partial_{t} u_{2}=\mathcal{J}_{2} \mathcal{L}\left(\llbracket \phi_{2} \rrbracket\right),
$$

and at $z=H$ :

$$
\begin{array}{ll}
\partial_{z} \phi_{1}=\partial_{z} \phi_{3}=0, & \phi_{3} \nu_{3}=\phi_{3} \partial_{t} h(t)-\mathcal{J}_{3} \mathcal{L}\left(\llbracket \phi_{3} \rrbracket\right), \\
\partial_{z} u_{1}=A_{1}\left(u_{1}-h(t)+h\left(t_{0}\right)\right), & \partial_{z} u_{2}=A_{2}\left(u_{2}-h(t)+h\left(t_{0}\right)\right),
\end{array}
$$

as they follow from (31), (32) and (35), respectively. We notice that we need in total 9 boundary conditions ( 2 for each of $\phi_{1}, \phi_{2}, u_{1}, u_{2}$ and 1 for $\left.v_{3}\right)$, as well as an extra condition to determine $h(t)$, so in total 10 conditions.

\subsection{Dimensionless formulation}

We nondimensionalize the fundamental variables, unknowns and parameters by dividing them by a normalization constant to make them dimensionless and $\mathcal{O}(1)$. The normalization constants are denoted as $U$ for the displacement, $H$ for the position, $V$ for the velocity, $T$ for the time, and $J$ for the flux. Material coefficients $\chi_{1,2}$ and $E_{1,2}$ are normalized with respect to the largest value of all constituents, so $\chi=\max \left\{\chi_{1}, \chi_{2}\right\}, E=\max \left\{E_{1}, E_{2}\right\}$. Moreover, we introduce the small parameter $\epsilon$ as the ratio of $U$ and $H$. This small parameter recalls that our model uses linear deformation theory, in which deformations are small with respect to the size of the domain. We note here that this assumption holds as long as $(h(t)-H) / H=\mathcal{O}(\epsilon)$. Concerning the choice of the time scale $T$, we have three natural options: diffusion time scale $T=U / V$, reaction time scale $T=1 / K$, and inflow time scale $T=U / J$. If we opt for the diffusion time scale and nondimensionalize Eq. (43c) making all terms and coefficients of the same order, we obtain $V=H K$ and $J=H K$ yielding $T=U / V=U / J=(U / H) / K=\epsilon / K$, for both the diffusion and the inflow time scale. Consequently, the diffusion time scale is much smaller than the reaction time scale, implying that diffusion is much faster than the reaction, and therefore we opt here for the normalization constant $T=U / V=\epsilon / K$. Analogously, we find from Eqs. (43d) or (43e) the relation $E U / H^{2}=\chi V$. All this leads to the definitions of the following dimensionless numbers, viz:

$V=H K, T=\epsilon / K, U=\frac{\chi H^{3} K}{E}, J=H K$, and $\epsilon=\frac{\chi H^{2} K}{E}$.

Looking at the problem at the diffusion time scale regime, we obtain the following nondimensionalized system equations:

$$
\begin{aligned}
\partial_{t} \phi_{1}+\epsilon \partial_{z}\left(\phi_{1} \partial_{t} u_{1}\right)-\epsilon \delta_{1} \partial_{z}^{2} \phi_{1}= & \epsilon \kappa_{1} \mathcal{F}\left(\phi_{1}, \phi_{3}\right), \\
\partial_{t} \phi_{3}+\epsilon \partial_{z}\left(\phi_{3} \nu_{3}\right)-\epsilon \delta_{3} \partial_{z}^{2} \phi_{3}= & -\epsilon \kappa_{3} \mathcal{F}\left(\phi_{1}, \phi_{3}\right), \\
\partial_{z}\left(\phi_{1} \partial_{t} u_{1}+\phi_{2} \partial_{t} u_{2}+\phi_{3} \nu_{3}\right)-\sum_{\alpha} \delta_{\alpha} \partial_{z}^{2} \phi_{\alpha}= & S_{K} \mathcal{F}\left(\phi_{1}, \phi_{3}\right), \\
\chi_{1} \partial_{t} u_{1}-E_{1} \partial_{z}^{2} u_{1}-\gamma_{11} \partial_{z}^{2} \partial_{t} u_{1}-\gamma_{12} \partial_{z}^{2} \partial_{t} u_{2}= & \chi_{1} \nu_{3}-\partial_{z}\left(\phi_{1} E_{1} \partial_{z} u_{1}\right. \\
& \left.+\phi_{1} E_{2} \partial_{z} u_{2}\right), \\
\chi_{2} \partial_{t} u_{2}-E_{2} \partial_{z}^{2} u_{2}-\gamma_{22} \partial_{z}^{2} \partial_{t} u_{2}-\gamma_{21} \partial_{z}^{2} \partial_{t} u_{1}= & \chi_{2} \nu_{3}-\partial_{z}\left(\phi_{2} E_{1} \partial_{z} u_{1}\right. \\
& \left.+\phi_{2} E_{2} \partial_{z} u_{2}\right),
\end{aligned}
$$

where

$$
\kappa_{\alpha}=\frac{\mathcal{M}_{\alpha}}{\tilde{\rho}_{\alpha}} \frac{\tilde{\rho}_{1}}{\mathcal{M}_{1}} \frac{\tilde{\rho}_{3}}{\mathcal{M}_{3}} \frac{k}{K} .
$$

In these equations all material coefficients are made dimensionless in the usual way and without changing their notation, meaning that: 
$\left\{\delta_{\alpha} \rightarrow \delta_{\alpha} / K H^{2}=\delta / K H^{2} \tilde{\rho}_{\alpha}, \chi_{\alpha} \rightarrow \chi_{\alpha} / \chi, E_{\alpha} \rightarrow E_{\alpha} / E, \gamma_{\alpha} \rightarrow \gamma_{\alpha} / \chi H^{2}\right\}$

Due to the nondimensionalization, the domain changes from $(0, H)$ to $(0,1)$. The initial conditions and most of the boundary conditions do not change their structure. Only the nonzero boundary conditions at the upper boundary (now at $z=1$ ) change due to the introduction of the dimensionless boundary displacement function $\mathcal{W}(t)=(h(t)-H) / \epsilon H$ such that $\mathcal{W}=\mathcal{O}(1)$. The nonhomogeneous boundary conditions at $z=1$ become

$$
\begin{aligned}
\phi_{3}\left(\partial_{t} \mathcal{W}(t)-v_{3}\right) & =\mathcal{J}_{3} \mathcal{L}\left(\llbracket \phi_{3} \rrbracket\right), \\
\partial_{z} u_{1} & =A_{1}\left(u_{1}-\mathcal{W}(t)\right), \\
\partial_{z} u_{2} & =A_{2}\left(u_{2}-\mathcal{W}(t)\right) .
\end{aligned}
$$

Integrating Eq. (43c) from $z=0$ to $z=1$, and using (47) and (48), we obtain a closed expression for $\mathcal{W}(t)$ for all $t>0$ in terms of influxes, the production term by the chemical reaction, and the mismatch of displacement at the boundary, viz.

$$
\begin{aligned}
\mathcal{W}(t)= & \int_{0}^{t}\left[\mathcal{J}_{2} \mathcal{L}\left(\llbracket \phi_{2}(0, s) \rrbracket\right)+S_{K} \int_{0}^{1} \mathcal{F}\left(\phi_{1}(z, s), \phi_{3}(z, s)\right) \mathrm{d} z\right. \\
& \left.+\mathcal{J}_{3} \mathcal{L}\left(\llbracket \phi_{3}(1, s) \rrbracket\right)-\sum_{\alpha=1}^{2} \frac{\phi_{\alpha}(1, s)}{A_{\alpha}} \partial_{t} \partial_{z} u_{\alpha}(1, s)\right] \mathrm{d} s .
\end{aligned}
$$

We note here that in the limiting case $A_{1,2} \downarrow 0$, as then also $\partial_{z} u_{1,2} \rightarrow 0$, the last term of Eq. (54) becomes undetermined. In this case we cannot use (53b) and (53c), which results in the following adapted relation for $\mathcal{W}(t)$ (derived in a way analogously to the derivation of Eq. (54))

$$
\begin{aligned}
\mathcal{W}(t)= & \int_{0}^{t} \frac{1}{\phi_{3}(1, s)}\left[\mathcal{J}_{2} \mathcal{L} \llbracket \phi_{2}(0, s) \rrbracket\right)+S_{K} \int_{0}^{1} \mathcal{F}\left(\phi_{1}(z, s), \phi_{3}(z, s)\right) \mathrm{d} z \\
& \left.+\mathcal{J}_{3} \mathcal{L}\left(\llbracket \phi_{3}(1, s) \rrbracket\right)-\sum_{\alpha=1}^{2} \phi_{\alpha}(1, s) \partial_{t} u_{\alpha}(1, s)\right] \mathrm{d} s .
\end{aligned}
$$

From both these results we conclude that the first two terms, the influxes with $\mathcal{J}_{2,3}$ being positive, yield a positive contribution to $\mathcal{W}(t)$ making the layer increase in thickness. Whether or not the third term has an increasing or decreasing effect depends on the sign of $S_{K}$; when, as in our case, $S_{K}=-1$, the chemical reaction does shrink the layer. At this moment, nothing specific can be said for the last term. However, our numerical results reveal that the effect of this term is always small. Thus, we can state that the domain of the layer only grows if the magnitude of the first two terms is greater than the third one. Hence, there is a competition effect here.
In Appendix A, a solution for System D has been obtained as a formal asymptotic expansion in $\epsilon$. The asymptotic expansion is formal as it is not a priori known whether or not this expansion is converging in $\epsilon$. The predictive power of a formal asymptotic expansion should not be underestimated, because there exist formal asymptotic expansions, which are diverging, but can be very accurate when only a truncated version of the expansion is used; see the example in Section 1.4.2 on pages 13 and 14 of [21]. This motivated us in the choice of the two $J$-parameters; see Table 1 .

\section{Numerical method}

In this section, we solve numerically the systems $\mathrm{A}, \mathrm{C}$ and $D$. We omit system $B$, because a viscoelastic term is needed to obtain a coercive system, such as in system A, for which we have proven the convergence of the timediscrete evolutions to the corresponding weak solution; see [40]. We expect that similar convergence results can be obtained for the systems $\mathrm{C}$ and $\mathrm{D}$, as they have a viscoelastic term similar to the one in system A. Also, when solving system D we exclude the Laplacian terms in Eq. (43c), or stated in another way: the numerical method uses $\delta_{\alpha}=0$ for (43c). This exclusion is justified by an order analysis of the terms of (43c) from the $\phi_{\alpha}$-solutions of (43a) and (43b), which states that $\sum_{\alpha=1}^{3} \delta_{\alpha} \Delta \phi_{\alpha}=\mathcal{O}(\sqrt{\epsilon} \mathcal{F})$.

Our code is called NewGypsum and it is based on a combination of MATLAB routines. We start off with a Rothe time discretization of the systems A, C and D, which linearizes the systems. Benefitting from the onedimensional-in-space formulation, solving the linear systems is done automatically by using the built-in boundary value problem (BVP) solvers of MATLAB, see bvp4c and bvp 5c; $[22,23]$. These solvers take a grid, a guess for the solution, and the BVP system as input. Then they automatically readjust the grid and interpolate the guess solution to obtain a starting point for the numerical scheme, controlling a certain error metric to determine the solution based on user-defined-convergence criteria.

The solver bvp4c is an implicit Runge-Kutta method using the 3-stage Lobatto IIIa formula with control on the residual [22]. The method is only applicable to linear Lipschitz systems [22]. Fortunately, systems A, C, and D can be shown to satisfy this condition within certain parameter constraints (which we will explain more thoroughly in the next section). For an easy guide in understanding and using bvp 4 c we recommend [36]. Moreover, [36] shows that boundary layer effects are well resolved by the bvp $4 \mathrm{C}$ solver.

The solver bvp5c is an implicit Runge-Kutta method using the 4-stage Lobatto IIIa formula with control on the true error [23]. The solver bvp5c is more precise than bvp $4 \mathrm{c}$, but it is also less versatile [23]. This does not pose a problem as our three systems A, C and D still satisfy the 
applicability conditions for bvp $5 \mathrm{c}$ and bvp $5 \mathrm{c}$ has similar capabilities in handling boundary layers as bvp $4 \mathrm{c}$ [23]. In our case the choice was made to use bvp $5 \mathrm{c}$ as it made our simulations about 27 times faster than when using bvp $4 \mathrm{c}$.

A more detailed explanation of our NewGypsum can be found in Section 2.4 of [40]. Moreover, in Appendix A one can find a validation of the NewGypsum routine with a Mathematica simulation of the asymptotic $\epsilon$-expansion solutions derived in the same appendix.

\section{Quest for realistic numerical behavior}

Even though our systems were derived based on first principles in terms of balance/conservation laws, this does not guarantee that all physical constraints are automatically satisfied for large variations in the model parameters. A solution is said to show realistic behavior if the following three constraints are satisfied within this framework:

1 The volume fractions should be nonnegative and less than one. From the mathematical analysis point of view we expect that system $A$ behaves poorly when volume fractions become very small. To outlaw this unwanted behavior a positive minimal value $\phi_{\min }$ is introduced, leading to the constraint

$$
0<\phi_{\min } \leq \phi_{\alpha}(t, z)<1
$$

for all $\alpha \in\{1,2,3\}$, for all $z \in(0,1)$, and for all $t \in\left(0, t_{f}\right)$.

2 A second condition is a demand on the upper bound for the velocity. Fast local deformations are allowed as long as the total contribution to the domain deformation is still small, the stresses remain low and the quasi-static approximation is not violated. Hence, it is natural to cap both the total velocity in the domain and the total spatial change of the velocity in the domain. This is reflected in the condition

$\left\|\nu_{3}\right\|_{L^{2}\left(t_{0}, t ; H^{1}(0,1)\right)}^{2}=\int_{0}^{t}\left[\int_{0}^{1}\left(v_{3}(s, z)^{2}+\left(\partial_{z} v_{3}(s, z)\right)^{2}\right) \mathrm{d} z\right] \mathrm{d} s<V^{2}$

for all $t \in\left(0, t_{f}\right)$.

3 The concrete layer has two boundaries that allow influx. Even though the chemical reaction itself is volume contractive, the combination of influx and chemical reactions must be volume expansive due to the porous nature of gypsum [28]. Hence, the height of the plate-layer must be a nondecreasing function:

$$
\partial_{t} h(t)=\epsilon \partial_{t} \mathcal{W}(t) \geq 0 \quad \text { for all } t \in\left(0, t_{f}\right) .
$$

Realistic behavior is defined as satisfying all three constraints Eqs. (56) to (58). We immediately stop a simulation when one of the three inequalities is violated.

We need a benchmark of our numerical program to test the numerical solutions for realistic behavior. For this we introduce a reference set of material constants. The values of these constants, and their dimensionless counterparts, dimensionalized with respect to the diffusion time scale, are listed in Table 1. The numerical evaluations use a time step $\Delta t$, the size of the time interval $t_{f}$, and a number of spatial subdivisions, $1 / \Delta z$. We choose fixed values $\Delta t=0.001, t_{f}=0.500$ and $1 / \Delta z=300$ for these parameters. In the remainder of this paper we implicitly use the parameter values of Table 1, whenever parameter values are not explicitly specified. A spatial-temporal analysis of our benchmark problem with the parameter values of Table 1 can be found in Section 2.6 of [40], showing that our benchmark simulation gives expected behaviour.

\subsection{Parameter dependence of found realistic behavior}

We aim to determine how the size of the realistic time interval, given in number of numerical iterations $N_{R}$, depends on the system parameters. Our definition of realistic behavior contains three constraints, see the beginning of Section 5, which can be numerically checked. We investigate the numerical simulation applied to systems A, $\mathrm{C}$ and $\mathrm{D}$ for a large parameter range, by changing specific parameters in Table 1. In this way our results even hold when experimental values with large uncertainties are used for the model parameters if these values with uncertainties remain in the probed region. Out of the 20 model parameters, we will only change specific parameters chosen on basis of their influence on the analytical bounds in the existence proof in [40]. When a bound in this existence proof contains a product of two parameters, then this parameter pair is chosen. All parameters are modified in a double exponential fashion such that large parameter ranges are investigated. Finally, the initial condition $\left(\phi_{10}, \phi_{20}, \phi_{30}\right)$ is chosen, because they immediately determine whether chemical reactions or influx do occur.

We have chosen to investigate the response of the model with respect to the following parameters and parameter tuples, because these parameters or combinations of parameters are either crucial for System D from a physical perspective or dominant in mathematically derived upper bounds in the existence proof in [40]:

$\left(\phi_{10}, \phi_{20}, \phi_{30}\right), \quad \delta, \quad \epsilon, \quad\left(\mathcal{J}_{2}, \phi_{2, \text { res }}\right)$ and $\left(A_{1}, \gamma_{1}\right)$.

The parameter pair $\left(A_{2}, \gamma_{2}\right)$ should be investigated as well. However, we chose to fix the ratios $A_{1} / A_{2}$ and $\gamma_{1} / \gamma_{2}$, because the dependence on $\left(A_{2}, \gamma_{2}\right)$ is expected to be similar to the dependence on $\left(A_{1}, \gamma_{1}\right)$. Similarly, we chose to fix the ratios $\mathcal{J}_{2} / \mathcal{J}_{3}$ and $\phi_{2, \text { res }} / \phi_{3, \text { res }}$. Moreover, if parameters are not mentioned to have special values, then these parameters are set to their standard values as listed in Table 1. 


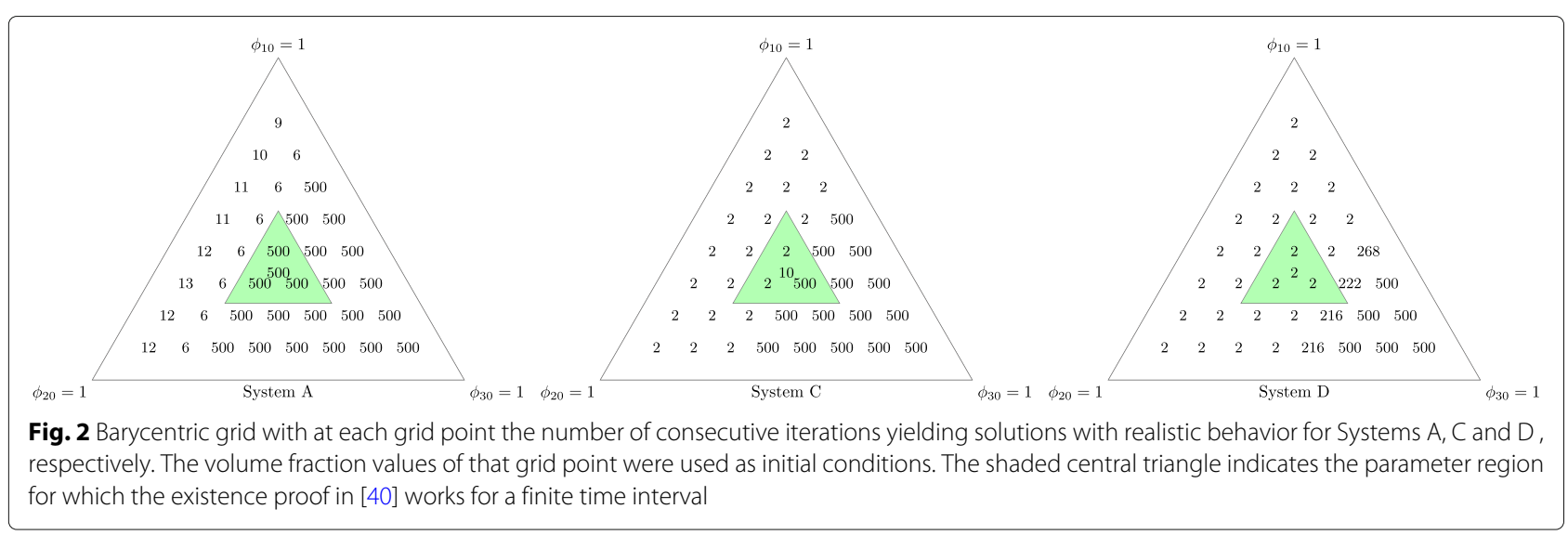

The existence proof in [40] points out a dependence on the $\left(\kappa_{1}, \kappa_{3}, \phi_{1, s a t}\right)$ parameter triple. However, the dependence on $\phi_{1 \text { sat }}, \kappa_{1}$ and $\kappa_{3}$ is quite subtle: only for $\phi_{1, \text { sat }}>$ $\phi_{1} \approx \phi_{10}$ the chemical reaction is active and $\mathcal{F}>0$. This has only a relevant effect on the incompressibility condition, because in the first two diffusion equations Eqs. (50a) and (50b) the right-hand sides are of $\mathcal{O}(\epsilon)$. This implies that the effect of $\kappa_{1}$ and $\kappa_{3}$ on the simulations is expected to be (negligibly) small. As we made not enough simulations for $\phi_{1}$ above the $\phi_{1, \text { sat }}$ threshold value, we can not draw any conclusions concerning its effect on realistic behavior. However, we expect an increasing $\phi_{1, \text { sat }}$ to decrease the size of the realistic time interval, as increasing $\phi_{1, \text { sat }}$ increases the size of $\mathcal{F}$ and, hence, also the size of $v_{3}$.

We investigate the triple $\left(\phi_{10}, \phi_{20}, \phi_{30}\right)$ using a barycentric triangular grid with grid size 0.1 , as shown in Fig. 2. The performance of the simulations is measured in terms of the number of consecutive iterations yielding realistic behavior. Each number denotes that the first unrealistic behavior occurs at the next iteration, while 500 denotes that no unrealistic behavior has been encountered. This performance value is placed at the grid point of the initial volume fraction values used for obtaining the result. We have added the existence region of [40] to the barycentric plots of Fig. 2 as a shaded region.

The three systems behave differently as one can see from the size of the parameter region with 500 iterations. The parametric region pointing at the high acid concentration region is outperforming the other parameter regions in all systems. A high concentration of acid implies that the reaction is slow (i.e. $\mathcal{F}$ is small), and consequently, the velocity $v_{3}$ remains small. Moreover, also the influx of acid is low or even absent. This results in a relatively small increase of the norm of $v_{3}$, and, therefore, violating the velocity norm upper bound (which is the most critical of the three conditions to violate) takes more time for large values of $\phi_{3}$. This explains the good performance of this parameter region.

For the determination of the dependence on other parameters the best choice of initial conditions for each system is exactly in the transition region between the regions of small (single digit) and high (500) amount of iterations. In this transition region, the amount of iterations is expected about half way in between 1 and 500 iterations. Any dependence yielding lower or higher amounts of iterations is faithfully represented. Outside this transition region the registration of the dependence is limited to a one-sided deviation of the reference level of amount of iterations, while in this transition region the registration allows for the full two-sided deviation of the reference level of the amount of iterations. We have chosen $\left(\phi_{10}, \phi_{20}, \phi_{30}\right)$ equal to $(11 / 30,11 / 30,8 / 30)$, $(1 / 3,1 / 3,1 / 3)$, and $(1 / 4,1 / 4,1 / 2)$ for System A, C, and D, respectively.

As for $\delta$ and $\epsilon$, we modified their values in an exponential fashion. Again, we recorded the amount of consecutive iterations, $N_{R}$, for which the solutions remained realistic. The amount $N_{R}$ for Systems A, C and D is recorded in Table 2 for changes in $\delta$.

For all systems, we see that the size of $\delta$ has practically no influence and is, therefore, unimportant in establishing

Table 2 Number of consecutive iterations yielding realistic behavior for Systems A, C and D at different values of $\delta$

\begin{tabular}{llllllllllll}
\hline & \multicolumn{1}{l}{$\delta=1.00 \times$ factor below } \\
\cline { 2 - 12 } & $10^{-5}$ & $10^{-4}$ & $10^{-3}$ & $10^{-2}$ & $10^{-1}$ & 1 & 10 & $10^{2}$ & $10^{3}$ & $10^{4}$ & $10^{5}$ \\
\hline A & 297 & 304 & 297 & 311 & 311 & 324 & 332 & 338 & 331 & 338 & 338 \\
C & 212 & 222 & 220 & 216 & 218 & 220 & 216 & 212 & 230 & 222 & 212 \\
D & 462 & 462 & 462 & 462 & 464 & 464 & 464 & 464 & 464 & 464 & 464 \\
\hline
\end{tabular}




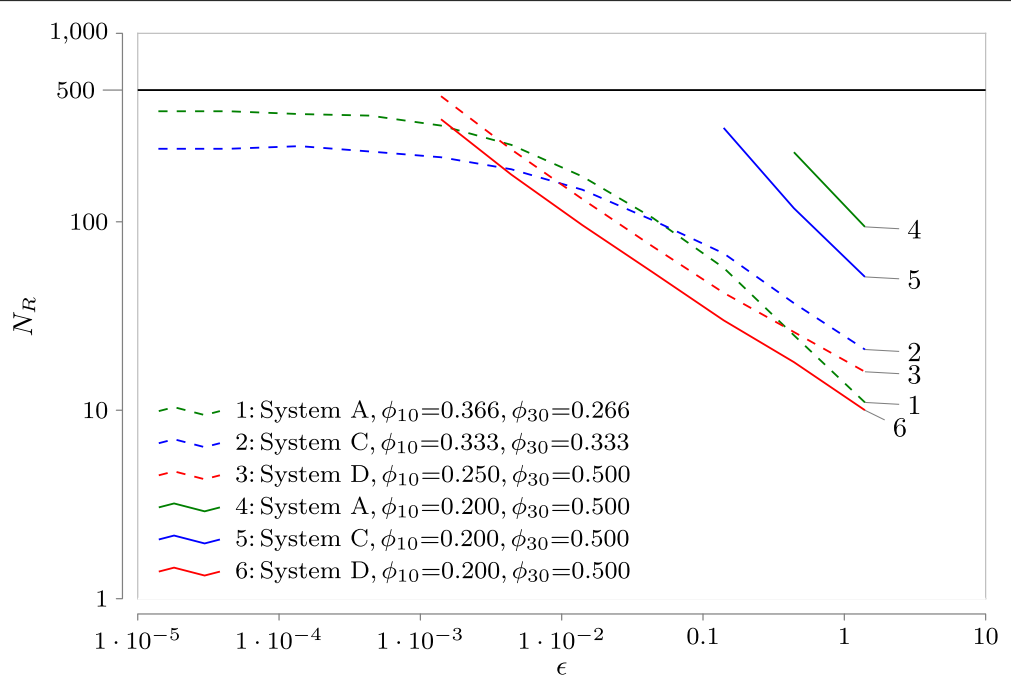

Fig. $3 \mathrm{Log}-$ log plot of the number of consecutive iterations yielding realistic behavior $\left(N_{R}\right)$ versus the parameter value of $\epsilon$ for several systems and initial values. Since the duration of the simulation was limited to 500 iterations, only the unambiguous values smaller than 500 iterations are chosen

realistic behavior defined in this section. This makes sense because the initial conditions are smooth, which leads to small values of the Laplacian. Hence, $\delta$ has only a minor effect on the simulation output.

In Fig. 3, the values of $N_{R}$ are plotted for systems A, $\mathrm{C}$ and $\mathrm{D}$ for $\epsilon$ equal to 1.4 times a factor equal to all powers of $\sqrt{10}$ between $10^{-2}$ and $10^{3}$. Only the unambiguous values of $N_{R}<500$, are plotted next to similar simulations executed with the modified parameter values $\left(\phi_{10}, \phi_{20}, \phi_{30}\right)=(0.2,0.3,0.5)$.

The effect of $\epsilon$ shows a different performance for $\epsilon \geq$ 0.0014 and $\epsilon<0.0014$, where in the former case the behavior becomes worse for greater values of $\epsilon$. However, one should be aware that only small values of $\epsilon$ are acceptable because our model is based on the assumption of linear (small) deformations $(\epsilon \ll 1)$. The linear behavior of system $D$ in the log-log plot of Fig. 3 is a clear power law signal. In Table 3, we have listed the power law exponent estimate and its unbiased variance estimate for both initial value data sets. The estimators are explained in detail in Section 14.2 of [34]. Essentially, $T_{\text {real }}=N_{R} \Delta t \sim \epsilon^{-0.5}$ is a reasonable hypothesis for System $\mathrm{D}$ and it indicates how the validity of our model depends on physical scale separation.

Table 3 Unbiased estimators of $\alpha_{0}$ and their standard error for the relationship $T_{\text {real }} \sim \epsilon^{\alpha_{0}}$ describing the dependence of the realistic time interval of System D on the parameter $\epsilon$ for two different initial conditions

\begin{tabular}{lll}
\hline System D: $\left(\phi_{10}, \phi_{30}\right)$ & $(0.20,0.50)$ & $(0.25,0.50)$ \\
\hline$\hat{\alpha}_{0}$ & -0.509 & -0.487 \\
${\text { S } \hat{\alpha}_{0}}^{\#}$ datapoints & 0.00854 & 0.0121 \\
\hline
\end{tabular}

The realistic behavior is affected by changes in $\mathcal{J}_{\alpha}$, $\alpha \in\{2,3\}$, as they control the rate of influx and so a major aspect of thickness growth. Increasing the size of $\mathcal{J}_{\alpha}$ gives a corresponding increase in the size of $\mathcal{W}(t)$ for large enough $\mathcal{J}_{\alpha}$. However, for small $\mathcal{J}_{\alpha}$ we cannot expect the same correspondence, because at some point the reaction becomes the dominant contributor. Hence, for small $\mathcal{J}_{\alpha}$ the growth of $\mathcal{W}(t)$ must be independent of $\mathcal{J}_{\alpha}$, while at large $\mathcal{J}_{\alpha}$ this growth must be in a one-to-one correspondence.

The size of $\mathcal{W}(t)$ correlates with the size of $\mathcal{J}_{\alpha}$, see Eq. (54). However, Eqs. (47) and (48) show that $\partial_{t} u_{2}$ and $v_{3}$ are related to $\mathcal{J}_{\alpha}$. The incompressibility condition Eq. (50c) immediately gives that the norm of $v_{3}$ is, then, correlated with the size of $\mathcal{J}_{\alpha}$. Hence, from Eqs. (50c) and (54) we expect for small $\mathcal{J}_{\alpha}$ no dependence between the realistic time interval $T_{\text {real }}$ and $\mathcal{J}_{\alpha}$. At large $\mathcal{J}_{\alpha}$, we expect an inverse dependence of the realistic time interval $T_{\text {real }}$ on $\mathcal{J}_{\alpha}$. In Fig. 4 the expected behavior is shown. This figure also shows that the choice of the system (A, C, or D) and the value of $\phi_{\alpha, r e s}$ has only a minor influence on the realistic behavior.

The size of the viscoelastic parameter $\gamma_{\alpha}$ has a major effect on the realistic behavior: when $\gamma_{\alpha}$ is too small, the system loses coercivity and the numerical program immediately terminates. This happens for all values of $\gamma_{\alpha}<0.005$. For large enough values of $\gamma_{\alpha}$ the system preserves ellipticity, resulting in stable realistic behavior; see Table 4 for System A, C, and D.

The realistic behavior depends also on $A_{\alpha}$. When $A_{\alpha}$ takes large values, then the coupling between $\mathcal{W}(t)$ and the displacements $u_{1}$ and $u_{2}$ becomes strong, leading to a larger value of $\nu_{3}$, and thus smaller $N_{R}$. On the other hand, when $A_{\alpha}$ is small (say $A_{\alpha}<1$ ), then the boundary 


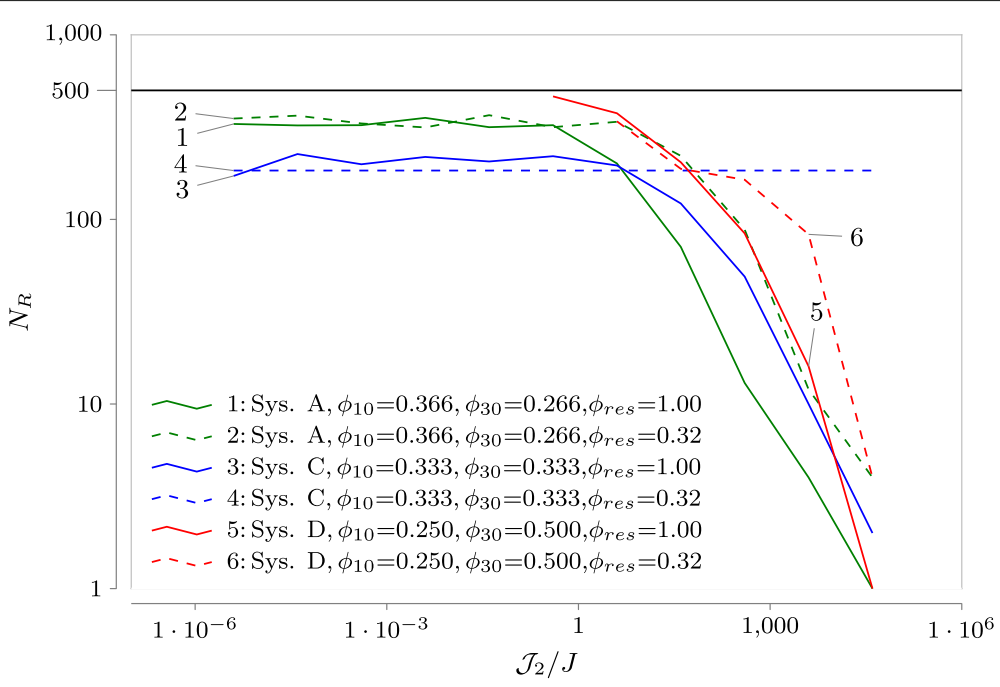

Fig. $4 \mathrm{Log}$-log plot of the number of consecutive iterations yielding realistic behavior $\left(N_{R}\right)$ versus the parameter value of $\mathcal{J}_{2}$ for all three systems at two different values for $\phi_{2, \text { res, }}$ with $\mathcal{J}_{3} / J=5 * \mathcal{J}_{2} / J$. Notice the two regions with different performance as expected due to the influence of $\mathcal{W}(t)$ on $v_{3}$

condition will behave more like a Neumann boundary condition, having no effect whatsoever on the realistic time interval. Again, we see these behaviors in Table 4 for Systems A, C, and D. This behavior agrees with the analytical results from [40] for System A.

For System D, we have used the standard values for the parameters and initial conditions, the dimensionless thickness growth $\mathcal{W}(t)$. In Fig. 5, the results for a set of $\epsilon$-values are depicted. For $\epsilon \geq 0.0014$ the curve of $\mathcal{W}(t)$ has a rotated $S$ shape, whereas for $\epsilon<0.0014$ the behavior is linear and identically the same for all $\epsilon$. This linear behavior is clearly different for $t / T \approx 0$ and should not be confused with a windowing artifact applied to an Sshaped curve as the linear behavior occurs immediately and does not show a characteristic decrease in slope as with $\epsilon<0.0014$. Therefore, it seems there exists a bifurcation value of $\epsilon$ at which the system changes the qualitative behavior in $\mathcal{W}(t)$ near $t=0$. A deeper insight in this aspect requires more numerical and theoretical investigations. Future investigations are needed to shed light on this bifurcation behavior.

\section{Conclusion}

We have derived, based on first principles, several models describing concrete corrosion by taking into account mixture theory, small deformations, compressibility and viscoelastic effects, diffusion, chemical reactions, influx of chemical species and an expanding domain. The most suitable model is System D. For this system, we could obtain the best numerical results with nice power law

Table 4 Number of consecutive iterations yielding realistic behavior $\left(N_{R}\right)$ for Systems $A, C$ and $D$, and a set of values for the parameter pair $\left(A_{1}, \gamma_{1}\right)$

\begin{tabular}{|c|c|c|c|c|c|c|c|c|c|c|c|c|}
\hline \multirow[b]{3}{*}{$2 A_{1}$} & \multicolumn{4}{|c|}{ System A } & \multicolumn{4}{|c|}{ System C } & \multicolumn{4}{|c|}{ System D } \\
\hline & \multicolumn{4}{|l|}{$2 \gamma_{1}$} & \multicolumn{4}{|l|}{$2 \gamma_{1}$} & \multicolumn{4}{|l|}{$2 \gamma_{1}$} \\
\hline & 0.01 & 0.1 & 1 & 10 & 0.01 & 0.1 & 1 & 10 & 0.01 & 0.1 & 1 & 10 \\
\hline $0.1^{5}$ & 12 & 13 & 57 & 500 & 16 & 17 & 33 & 500 & 410 & 410 & 410 & 412 \\
\hline $0.1^{4}$ & 12 & 13 & 57 & 500 & 16 & 17 & 33 & 500 & 410 & 410 & 410 & 412 \\
\hline $0.1^{3}$ & 12 & 13 & 59 & 500 & 16 & 17 & 33 & 500 & 410 & 410 & 410 & 412 \\
\hline $0.1^{2}$ & 12 & 13 & 59 & 500 & 14 & 17 & 33 & 500 & 410 & 410 & 412 & 412 \\
\hline 0.1 & 10 & 14 & 123 & 500 & 14 & 19 & 38 & 500 & 416 & 416 & 416 & 418 \\
\hline 1 & 8 & 10 & 324 & 1 & 12 & 14 & 220 & 45 & 462 & 464 & 464 & 464 \\
\hline 10 & 8 & 1 & 4 & 6 & 10 & 1 & 4 & 8 & 244 & 320 & 308 & 306 \\
\hline $10^{2}$ & 2 & 6 & 8 & 8 & 2 & 4 & 8 & 8 & 1 & 1 & 1 & 1 \\
\hline
\end{tabular}

The values for $2 \gamma_{1} \leq 0.1^{3}$ were omitted since the system lost coercivity and therefore no simulation was performed. The values for $\gamma_{1}=50$ have been omitted for brevity since they are almost identical to the values for $\gamma_{1}=5$ 


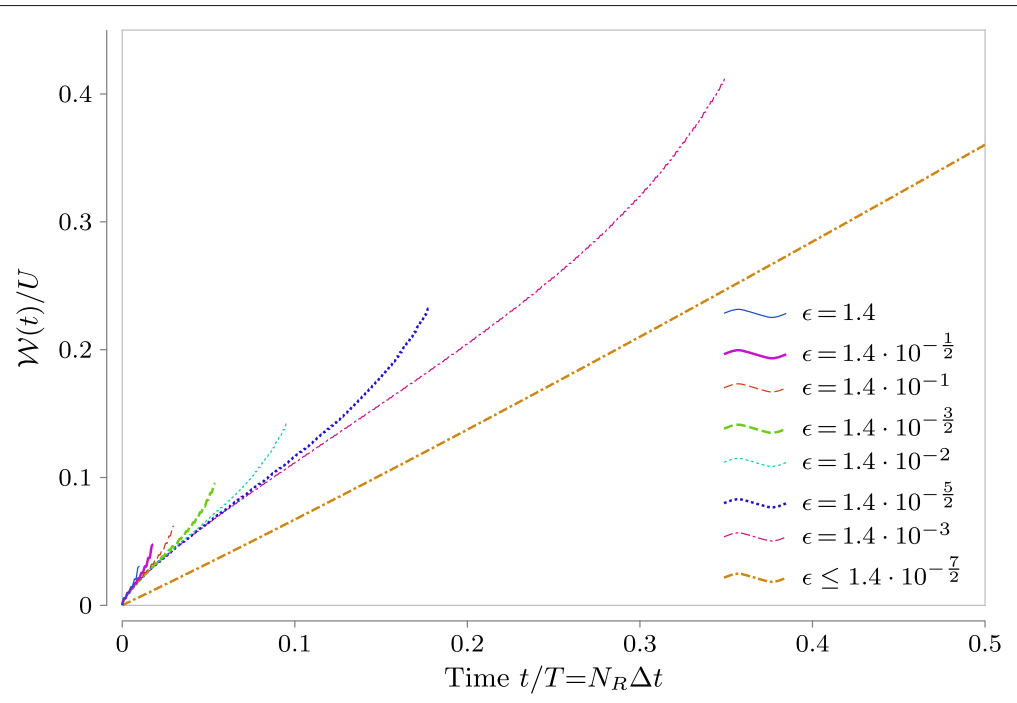

Fig. 5 A plot of $\mathcal{W}(t)$ in System $D$ at different parameter values of $\epsilon$

behaviors, which lead us to the hypothesis that the realistic time interval $T_{\text {real }}$ scales as $1 / \sqrt{\epsilon}$. Moreover, we could interpret the spatial behavior of all variables by taking into account the physical effects of the chemical reaction and of the influx of reacting materials.

Even though we have derived our systems from first principles, many material constants $\left(\delta_{\alpha}, \gamma_{\alpha}, A_{\alpha}\right)$ have either unknown values or are determined at length scales orders of magnitude larger than our simulated domain (e.g. the Young modulus cf. [32]). Consequently, many of our model parameters need to be identified. Better insight in the model parameters is needed. This can be obtained in at least three ways:

(i) By performing more specific measurements at the length scale of our domain;

(ii) By upscaling procedures, obtain effective material coefficients at length scales compatible with the measurements;

(iii) By suitably combining (i) and (ii).

By performing simulations with intentionally large parameter ranges, we localized the uncertainties in the model parameters and probed simultaneously the continuous dependence of the solution to our systems on the choice of parameters. In this way, the behavior of System $D$ is valid, even for the model parameters with large uncertainties. While probing the parameter dependence of our system on 20 different parameters, of which about 10 are indeterminate, we immediately encounter the curse of dimensionality - sampling a high dimensional space ${ }^{2}$ is a sparse operation. A more structured sampling was possible by targeting the variables present in analytical upper bounds derived in [40]. An additional complication is the nonlinear coupling of all unknowns involved concurrently in several physical processes. Such a strong coupling prohibits a fast simulation at a single parameter tuple and creates a complex nonlinear parameter dependence of the solution behavior.

What concerns System D, at least for a short transient time the realistic behavior showed practically constant concentrations due to the slow reaction with respect to the influx. The displacements and velocities seemed consistent with the influx of material, while the thickness of the concrete layer was growing steadily, as expected from real world observations. Moreover, these results coincide with [4] as the plate thickness increases in time and the correct changes in volume fractions were observed. Displacements and velocities could not be related to any quantity in [4], because their reaction occurs in the boundary, while ours occurs in the full domain.

The Systems A, C, and D showed strong dependence on several parameters. For all systems the number of consecutive iterations yielding realistic behavior $\left(N_{R}\right)$ is highly dependent on the choice of $\phi_{30}$, due to the incompressibility condition, while $\phi_{10}$ and $\phi_{20}$ seem unimportant, as long as $\phi_{10}+\phi_{20}=1-\phi_{30}$. The diffusion coefficient $\delta>0$ had no effect on $N_{R}$, while the scale separation parameter $\epsilon$ greatly influenced $N_{R}$ for all systems, especially for System D with an apparent power law dependence. The reaction parameters $\kappa_{1}, \kappa_{3}, \phi_{1, \text { sat }}$ had no influence on $N_{R}$, because $\epsilon$ is small and $\mathcal{J}_{3}>1$. The flux parameters $\mathcal{J}_{2}$ and $\mathcal{J}_{3}$ are unimportant at small values $\left(\mathcal{J}_{2}<1\right)$, while almost in one to one correspondence with $N_{R}$ at large values $\left(\mathcal{J}_{2}>10\right)$ due to Eq. (54). The external concentrations $\phi_{\text {res }}$ had almost no influence on $N_{R}$, what can be attributed to an under sampling of large values ( $\phi_{\text {res }}>0.3$ ). The viscoelastic parameters $\gamma_{1}$ and $\gamma_{2}$ are important for keeping coercivity. They show a high dependence on $N_{R}$ 
for Systems A and $\mathrm{C}$, but almost no dependence for System D. The boundary condition parameters $A_{1}$ and $A_{2}$ highly influence $N_{R}$, but for Systems A and $C$ the behavior seems erratic, except at small values due to the convergence to Neumann boundary conditions. The thickness $\mathcal{W}(t)$ for System $\mathrm{D}$ becomes larger for smaller values of $\epsilon$, but changes behavior for $\epsilon<0.0014$, for which $\mathcal{W}(t)$ seems independent of $\epsilon$. This behavioral change is unexpected and advocates for additional research. Moreover, the thickness $\mathcal{W}(t)$ increases continuously as expected from experiments.

Hence, the important parameters of Systems A, C, and $\mathrm{D}$ describing the behavior of $N_{R}$ are $\phi_{30}, \epsilon, \mathcal{J}_{\alpha}, \gamma_{\beta}$ and $A_{\beta}$ for $\alpha \in\{2,3\}$ and $\beta \in\{1,2\}$. Moreover, the observed behavior of the thickness $\mathcal{W}(t)$ is largely as expected from observations.

\section{Endnotes}

${ }^{1}$ In principle the right-hand side of Eq. (31) should be $-\phi_{\alpha}\left(\mathbf{v}_{\alpha}-\mathbf{V}\right)$ instead of 0 . However, in our linear theory the value 0 is justified due to the scale separation between displacement and the actual size of the domain. See Section 3 for the effect of scale separation on the system in the dimension reduction process.

${ }^{2}$ In our case, the dimensionality is linked to the space of simulations for all possible combinations of parameter values.

\section{Appendix A}

\section{Asymptotic $\boldsymbol{\varepsilon}$-small solutions to System D}

The system (50a)-(50e) contains the small parameter $\epsilon, 0<\epsilon \ll 1$, and we assume that the solution of this system can be expanded as a Poincaré series in $\epsilon$, for instance:

$$
\phi_{\alpha}(z, t ; \epsilon)=\phi_{\alpha}^{(0)}(z, t)+\epsilon \phi_{\alpha}^{(1)}(z, t)+\ldots,
$$

and the same for $\mathbf{u}_{\alpha}(z, t ; \epsilon)$ and $v_{3}(z, t ; \epsilon)$.

We substitute these expansions into the equations of system (50a)-(50e) and develop them with respect to $\epsilon$. We start with (50a), which results in

$$
\begin{aligned}
\partial_{t} \phi_{1}^{(0)} & +\epsilon\left(\partial_{t} \phi_{1}^{(1)}+\partial_{z}\left(\phi_{1}^{(0)} \partial_{t} u_{1}^{(0)}\right)-\delta_{1} \partial_{z}^{2} \phi_{1}^{(0)}-\kappa_{1} \mathcal{F}\left(\phi_{1}^{(0)}, \phi_{3}^{(0)}\right)\right) \\
& +O\left(\epsilon^{2}\right)=0 .
\end{aligned}
$$

The $\epsilon^{0}$-term yields $\partial_{t} \phi_{1}^{(0)}=0$. Together with the initial condition $\phi_{1}^{(0)}(z, 0)=\phi_{10}$, this gives $\phi_{1}^{(0)}(z, t)=\phi_{10}$ for all $t>0$ and all $z \in(0,1)$. If needed, the following equation for the first-order perturbation of $\phi_{1}$ can be used

$$
\partial_{t} \phi_{1}^{(1)}+\phi_{10} \partial_{z} \partial_{t} u_{1}^{(0)}=\kappa_{1} \mathcal{F}\left(\phi_{10}, \phi_{30}\right)=: \kappa_{1} F_{0} .
$$

In analogous way we obtain from (50b): $\phi_{3}^{(0)}(z, t)=\phi_{30}$, implying that also $\phi_{2}^{(0)}(z, t)=1-\phi_{10}-\phi_{30}=\phi_{20}$, and

$$
\partial_{t} \phi_{3}^{(1)}+\phi_{30} \partial_{z} v_{3}^{(0)}=-\kappa_{3} F_{0} .
$$

For the remaining three Eqs. (50c), (50d), and (50e), we are only interested in the zeroth-order approximation, meaning that we let $\epsilon \rightarrow 0$. From here on, we denote $u_{1}^{(0)}, u_{2}^{(0)}, v_{3}^{(0)}$ simply by $u_{1}, u_{2}, v_{3}$; moreover we use here $S_{K}=-1$. This reduces these equations to:

$$
\begin{aligned}
\partial_{z}\left(\phi_{10} \partial_{t} u_{1}+\phi_{20} \partial_{t} u_{2}+\phi_{30} v_{3}\right) & =-F_{0}, \\
\chi_{1} \partial_{t} u_{1}-\left(1-\phi_{10}\right) E_{1} \partial_{z}^{2} u_{1}+\phi_{10} E_{2} \partial_{z}^{2} u_{2}-\gamma \partial_{z}^{2} \partial_{t}\left(u_{1}-u_{2}\right) & =\chi_{1} v_{3}, \\
\chi_{2} \partial_{t} u_{2}+\phi_{20} E_{1} \partial_{z}^{2} u_{1}-\left(1-\phi_{20}\right) E_{2} \partial_{z}^{2} u_{2}-\gamma \partial_{z}^{2} \partial_{t}\left(u_{2}-u_{1}\right) & =\chi_{2} v_{3} .
\end{aligned}
$$

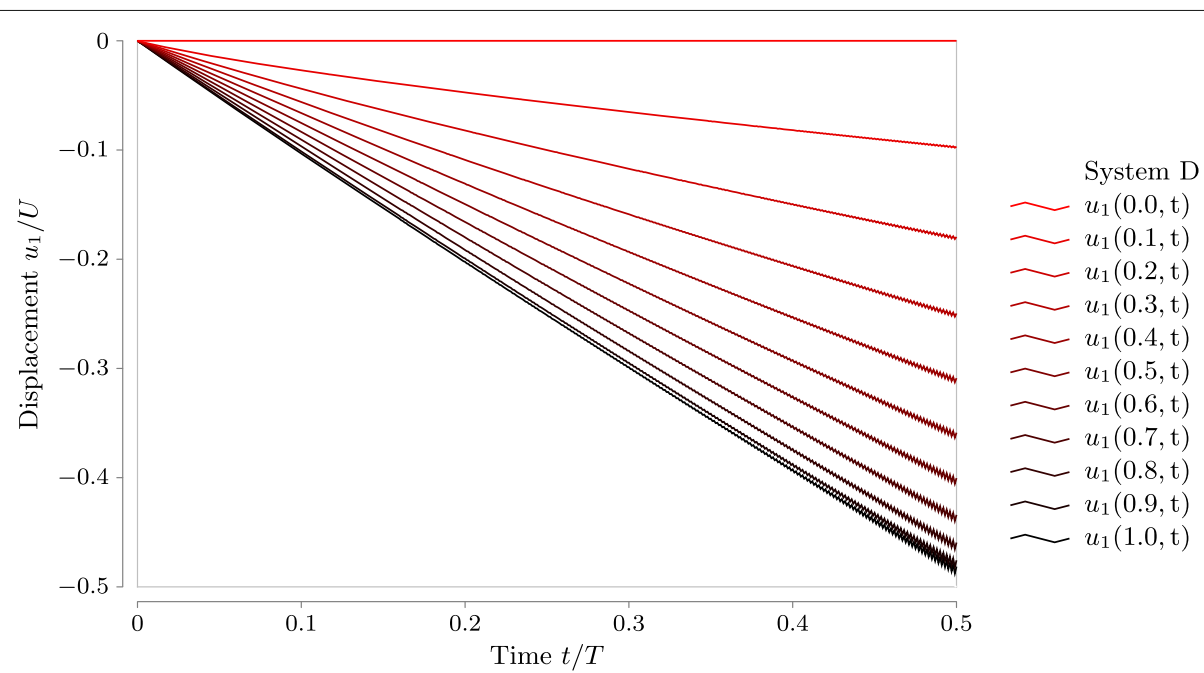

Fig. 6 MATLAB simulation of $u_{1}(z, t)$ for $z \in\{0,0.1, \ldots, 0.9,1\}$ for $A_{1}=A_{2}=0$ and the other parameters with the values of Table 1 . The oscillations in the graphs are due to an unphysical alternating-in-time solution of $v_{3}(z, t)$ in the MATLAB simulation 


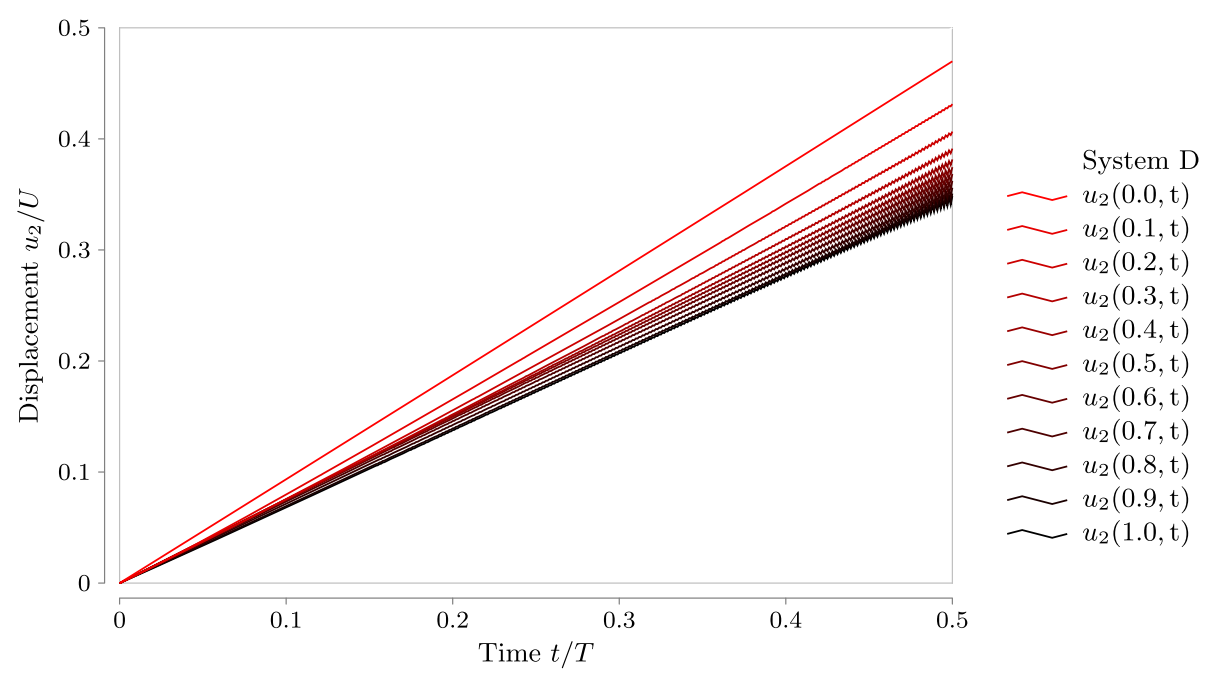

Fig. 7 MATLAB simulation of $u_{2}(z, t)$ for $z \in\{0,0.1, \ldots, 0.9,1\}$ for $A_{1}=A_{2}=0$ and the other parameters with the values of Table 1 . The oscillations in the graphs are due to an unphysical alternating-in-time solution of $v_{3}(z, t)$ in the MATLAB simulation

Integrating the first equation to $z$ and using the boundary condition at $z=0: \phi_{20} \partial_{t} u_{2}(0, t)=\mathcal{J}_{2} \mathcal{L}\left(\phi_{2, \text { res }}-\phi_{20}\right)=: F_{1}$, we obtain

$$
v_{3}=\frac{1}{\phi_{30}}\left(F_{1}-F_{0} z-\phi_{10} \partial_{t} u_{1}-\phi_{20} \partial_{t} u_{2}\right)
$$

We eliminate $v_{3}$ with use of this relation from the last two equations. After some manipulations we can write these two equations as one matrix equation of the form

$$
\mathbb{A} \partial_{t} \mathbf{u}-\mathbb{B} \partial_{z}^{2} \mathbf{u}-\mathbb{C} \partial_{z}^{2} \partial_{t} \mathbf{u}=\mathbf{r}
$$

with

$$
\begin{aligned}
\mathbf{u} & =\left[\begin{array}{l}
u_{1} \\
u_{2}
\end{array}\right] \\
\mathbb{A} & =\frac{1}{\phi_{30}}\left[\begin{array}{cc}
\left(\phi_{10}+\phi_{30}\right) \chi_{1} & \phi_{20} \chi_{1} \\
\phi_{10} \chi_{2} & \left(\phi_{20}+\phi_{30}\right) \chi_{2}
\end{array}\right] \\
\mathbb{B} & =\left[\begin{array}{cc}
\left(\phi_{20}+\phi_{30}\right) E_{1} & -\phi_{10} E_{2} \\
-\phi_{20} E_{1} & \left(\phi_{10}+\phi_{30}\right) E_{2}
\end{array}\right] \\
\mathbb{C} & =\left[\begin{array}{cc}
\gamma & -\gamma \\
-\gamma & \gamma
\end{array}\right] \\
\mathbf{r}=\mathbf{r}(z) & =\frac{F_{1}-F_{0} z}{\phi_{30}}\left[\begin{array}{l}
\chi_{1} \\
\chi_{2}
\end{array}\right]
\end{aligned}
$$

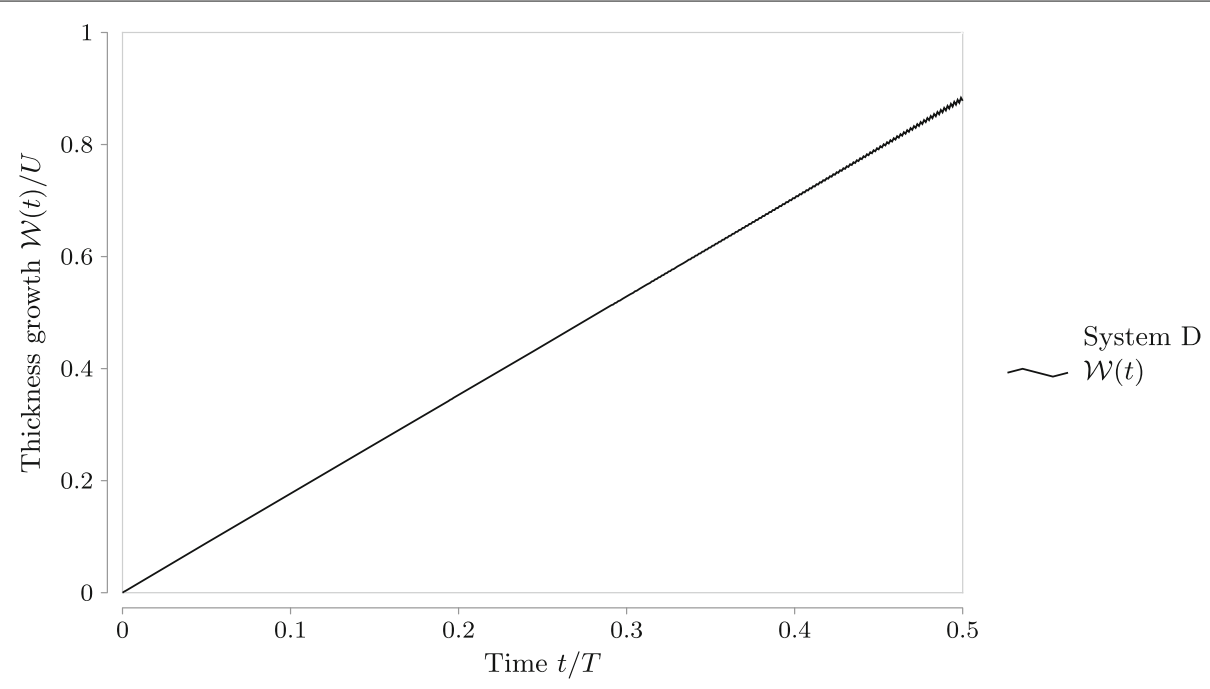

Fig. 8 MATLAB simulation of $\mathcal{W}(t)$ for $A_{1}=A_{2}=0$ and the other parameters with the values of Table 1 . The oscillations in the graphs are due to an unphysical alternating-in-time solution of $v_{3}(z, t)$ in the MATLAB simulation 


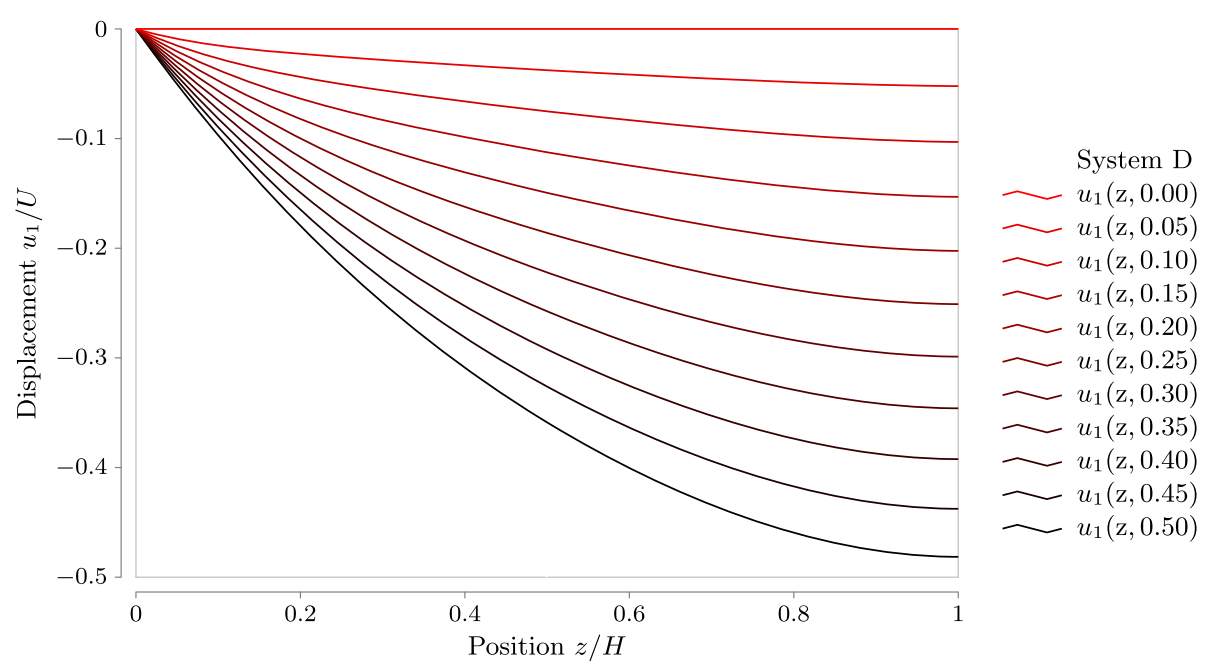

Fig. 9 MATLAB simulation of $u_{1}(z, t)$ for $t \in\{0,0.05, \ldots, 0.45,0.50\}$ for $A_{1}=A_{2}=0$ and the other parameters with the values of Table 1 . The oscillations in the graphs are due to an unphysical alternating-in-time solution of $v_{3}(z, t)$ in the MATLAB simulation

This system is a linear pseudo-parabolic system with constant coefficients for 2 unknown variables: $u_{1}(z, t)$ and $u_{2}(z, t)$ and for $z \in(0,1)$ and $t \in\left(0, t_{f}\right)$. The initial and boundary conditions for this system are $\mathbf{u}(z, 0)=\mathbf{0}$ and

$$
\begin{array}{rrr}
\text { at } z=0, & \mathbf{u}(0, t)=\mathbf{J}= & \left\{0, F_{1} / \phi_{20}\right\}, \\
\text { at } z=1, & \partial_{z} \mathbf{u}(1, t) & =\mathbf{0} .
\end{array}
$$

Moreover, $\mathcal{W}(t)$ can be found from (55) as

$\mathcal{W}(t)=\left(F_{1}+\phi_{30} J_{3}-F_{0}\right) t-\phi_{10} u_{1}(1, t)-\phi_{20} u_{2}(1, t)$

with $\phi_{30} J_{3}:=\mathcal{J}_{3} \mathcal{L}\left(\phi_{3, \text { res }}-\phi_{30}\right)$.
For $\chi_{1} \chi_{2} \phi_{30} \neq 0$ and $E_{1} E_{2} \phi_{30} \neq 0$, we can rewrite the pseudo-parabolic equation above as an initial-boundaryvalue problem by introducing

$$
\mathbf{u}(z, t)=\mathbf{U}_{0}(z)+\mathbf{J} t+\tilde{\mathbf{u}}(z, t),
$$

where the first two terms are chosen such that $\tilde{\mathbf{u}}$ satisfies the homogeneous pseudo-parabolic equation

$\mathcal{D}_{\mathrm{PP}}\{\tilde{\mathbf{u}}\}=\partial_{t} \tilde{\mathbf{u}}(z, t)-\hat{\mathbb{B}} \partial_{z}^{2} \tilde{\mathbf{u}}(z, t)-\hat{\mathbb{C}} \partial_{z}^{2} \partial_{t} \tilde{\mathbf{u}}(z, t)=\mathbf{0}$,

together with the homogeneous boundary conditions

$$
\begin{aligned}
\text { at } z & =0, & \tilde{\mathbf{u}}(0, t) & =\mathbf{0}, \\
\text { at } z & =1, & \partial_{z} \tilde{\mathbf{u}}(1, t) & =\mathbf{0},
\end{aligned}
$$

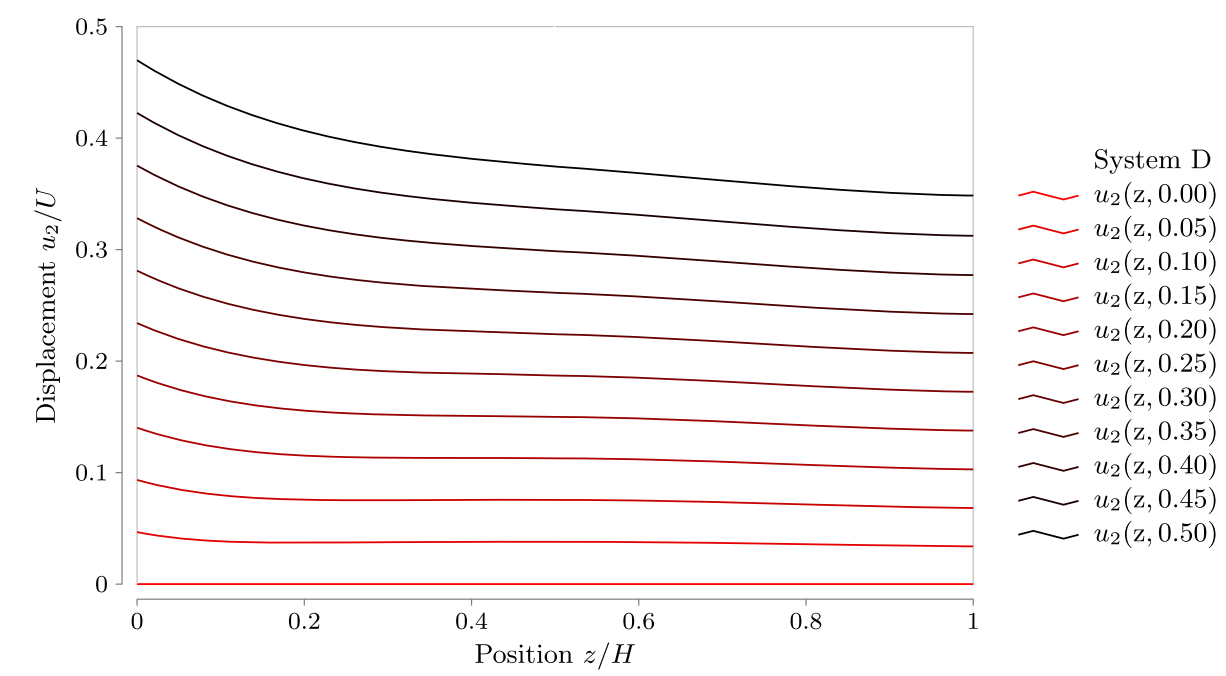

Fig. 10 MATLAB simulation of $u_{2}(z, t)$ for $t \in\{0,0.05, \ldots, 0.45,0.50\}$ for $A_{1}=A_{2}=0$ and the other parameters with the values of Table 1 . The oscillations in the graphs are due to an unphysical alternating-in-time solution of $v_{3}(z, t)$ in the MATLAB simulation 


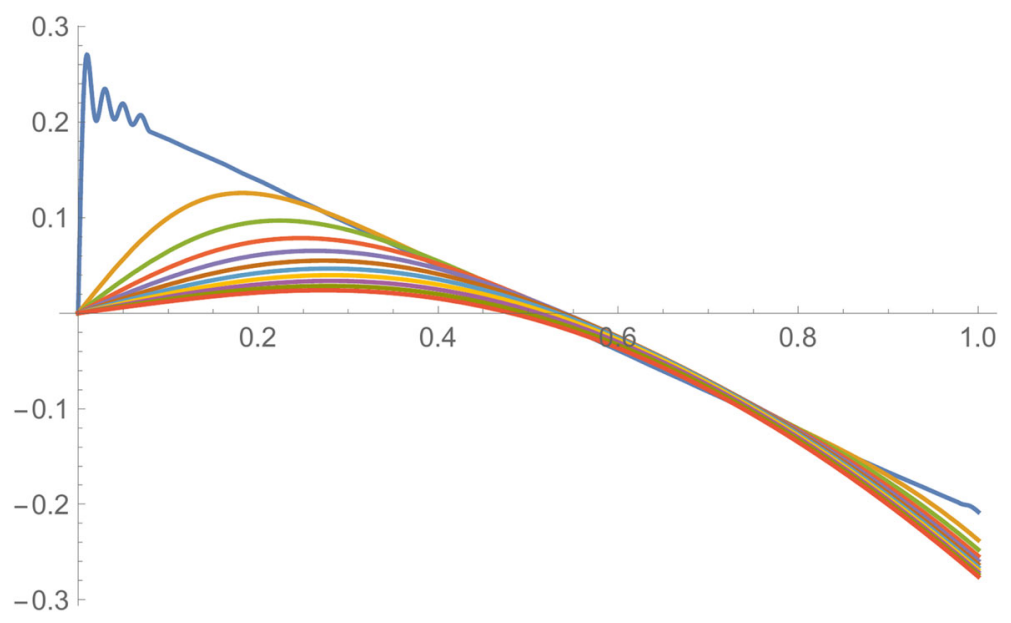

Fig. 11 Mathematica simulation of $v_{3}(z, t)$ with from top to bottom $t \in\{0,0.05, \ldots, 0.45,0.50\}$, respectively, for $A_{1}=A_{2}=0$ and the other parameters with the values of Table 1. The oscillatons in the $t=0$ graph are artifacts of the unevitable truncation of the infinite sum in Eq. (82)

and the inhomogeneous initial condition

$$
\tilde{\mathbf{u}}(z, 0)=-\mathbf{U}_{0}(z),
$$

such that the original initial condition $\mathbf{u}(z, 0)=\mathbf{0}$ is still satisfied. In (71) $\hat{\mathbb{B}}=\mathbb{A}^{-1} \mathbb{B}$ and $\hat{\mathbb{C}}=\mathbb{A}^{-1} \mathbb{C}$, and , while $\mathbf{U}_{0}(z)$ is given by

$$
\mathbf{U}_{0}(z)=\mathbf{b}_{1} z+\mathbf{b}_{2} z^{2}+\mathbf{b}_{3} z^{3},
$$

with $\mathbf{b}_{1}=-2 \mathbf{b}_{2}-3 \mathbf{b}_{3}, 2 \mathbf{b}_{2}=\hat{\mathbb{B}}^{-1}\left(\mathbf{J}-\hat{\mathbf{r}}_{1}\right)$ and $6 \mathbf{b}_{3}=-$ $\hat{\mathbb{B}}^{-1} \hat{\mathbf{r}}_{0}$, where $\hat{\mathbf{r}}(z)=\mathbb{A}^{-1} \mathbf{r}(z)=: \hat{\mathbf{r}}_{1}+\hat{\mathbf{r}}_{0} z$. Note, $\mathbb{A}$ and $\mathbb{B}$ are invertible because $\chi_{1} \chi_{2} \phi_{30} \neq 0$ and $E_{1} E_{2} \phi_{30} \neq 0$, respectively.

For $\gamma \neq-\frac{\chi_{1} \chi_{2}}{\chi_{1}+\chi_{2}} \frac{4}{\pi^{2}(2 k-1)^{2}}$ with $k \geq 1$ integer, i.e. $\gamma>0$ for $\chi_{1}, \chi_{2}>0$, we write the solution of (71) with the homogeneous boundary conditions as a series expansion in sine terms such that the boundary conditions are automatically satisfied of the form

$$
\tilde{\mathbf{u}}(z, t)=\sum_{k=1}^{\infty} \mathbf{U}_{k}(t) \sin \left(\zeta_{k} z\right),
$$

with $\zeta_{k}=(2 k-1) \pi / 2$, while the functions $\mathbf{U}_{k}(t)$ have to satisfy the ODE

$$
\partial_{t} \mathbf{U}_{k}(t)+\zeta_{k}^{2}\left(\hat{\mathbb{B}} \mathbf{U}_{k}(t)+\hat{\mathbb{C}} \partial_{t} \mathbf{U}_{k}(t)\right)=\mathbf{0},
$$

or, because $\hat{\mathbb{C}}+\zeta_{k}^{-2} \mathbb{I}$ is invertible due to choice of $\gamma$, slightly rewritten as

$$
\partial_{t} \mathbf{U}_{k}(t)+\mathbb{K}_{k} \mathbf{U}_{k}(t)=\mathbf{0},
$$

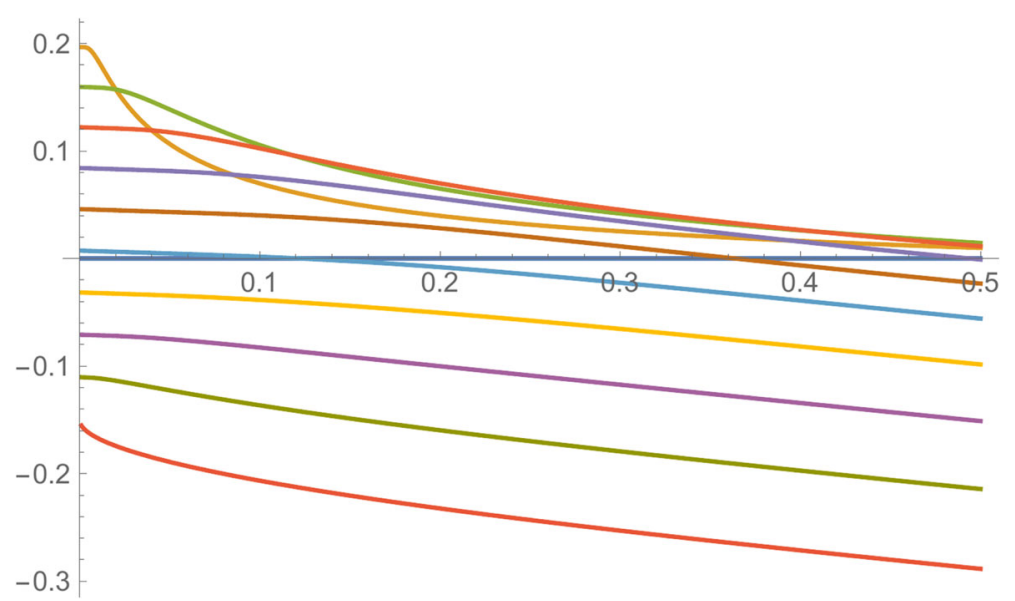

Fig. 12 Mathematica simulation of $v_{3}(z, t)$ for $z \in\{0,0.1, \ldots, 0.9,1\}$ with the order in the same color scheme as in Fig. 11 for $A_{1}=A_{2}=0$ and the other parameters with the values of Table 1 
with $\mathbb{K}_{k}=\left(\hat{\mathbb{C}}+\zeta_{k}^{-2} \mathbb{I}\right)^{-1} \hat{\mathbb{B}}$ and $\mathbb{I}$ the $2-\mathrm{D}$ unit matrix. This ODE has the fundamental solutions $\mathrm{e}^{-\lambda_{1 k} t}$ and $\mathrm{e}^{-\lambda_{2 k} t}$, where $\lambda_{1 k}$ and $\lambda_{2 k}$ are the eigenvalues of the $2 \times 2$-matrix $\mathbb{K}_{k}$. Hence, $\mathbf{U}_{k}(t)$ must be of the form

$$
\mathbf{U}_{k}(t)=\mathbb{U}_{k} \mathbf{C}_{k}(t),
$$

where $\mathbb{U}_{k}$ is the matrix of the eigenvectors of $\mathbb{K}_{k}$, i.e.

$$
\mathbb{U}_{k}=\left\{\left\{K_{k 12}, K_{k 12}\right\},\left\{\lambda_{1 k}-K_{k 11}, \lambda_{2 k}-K_{k 11}\right\}\right\},
$$

with $K_{k i j}$ the $(i, j)$ entry of $\mathbb{K}_{k}$, while

$$
\mathrm{C}_{k}(t)=\left\{c_{k 1} \mathrm{e}^{-\lambda_{1 k} t}, c_{k 2} \mathrm{e}^{-\lambda_{2 k} t}\right\},
$$

with $c_{k 1}$ and $c_{k 2}$ two unknown constants that will be determined from the condition that

$$
\begin{aligned}
\tilde{\mathbf{u}}(z, 0) & =\sum_{k=1}^{\infty} \mathbb{U}_{k} \mathbf{c}_{\mathbf{k}} \sin \left(\zeta_{k} z\right)=-\mathbf{U}_{0}(z), \\
\mathbf{c}_{\mathbf{k}} & =\mathbf{C}_{\mathbf{k}}(0)=\left\{c_{k 1}, c_{k 2}\right\} .
\end{aligned}
$$

Realizing that $\mathbf{U}_{0}(z)$ can be expanded in the sine series

$$
\begin{aligned}
\mathbf{U}_{0}(z) & =-\left(2 z-z^{2}\right) \mathbf{b}_{2}-\left(3 z-z^{3}\right) \\
\mathbf{b}_{3} & =S_{1}(z) \mathbf{b}_{2}+S_{2}(z) \mathbf{b}_{3}=: \sum_{k=1}^{\infty} \mathbf{B}_{k} \sin \left(\zeta_{k} z\right),
\end{aligned}
$$

with

$$
\begin{aligned}
& S_{1}(z)=-\frac{32}{\pi^{3}} \sum_{k=1}^{\infty} \frac{1}{(2 k-1)^{3}} \sin \left(\zeta_{k} z\right), \\
& S_{2}(z)=\frac{192}{\pi^{4}} \sum_{k=1}^{\infty} \frac{(-1)^{k}}{(2 k-1)^{4}} \sin \left(\zeta_{k} z\right),
\end{aligned}
$$

we find

$$
\mathbf{c}_{k}=-\mathbb{U}_{k}^{-1} \mathbf{B}_{k} \text {. }
$$

With this result, the solution for $\mathbf{u}(z, t)$ is complete. Recapitulating, we write (70) as

$$
\begin{aligned}
\mathbf{u}(z, t)= & -\left(2 z-z^{2}\right) \mathbf{b}_{2}-\left(3 z-z^{3}\right) \mathbf{b}_{3} \\
& +\mathbf{J} t+\sum_{k=1}^{\infty} \mathbb{U}_{k} \mathbf{C}_{k}(t) \sin \left(\zeta_{k} z\right) .
\end{aligned}
$$

Finally, we find $v_{3}(z, t)$ from (65) and $\mathcal{W}(t)$ from (69).

Simulating these results with both Mathematica and MATLAB gave near identical results, except for an unphysical velocity $v_{3}(z, t)$ in the MATLAB simulation yielding almost negligible small oscillations in time for $u_{1}(z, t), u_{2}(z, t)$, and $\mathcal{W}(z, t)$. Even though the MATLAB and Mathematica simulations use different approaches, especially for determining the initial velocity $v_{3}\left(z, 0^{+}\right)$, we can conclude that both simulations are accurate with respect to $u_{1}(z, t), u_{2}(z, t)$, and $\mathcal{W}(z, t)$, while only the Mathematica simulation shows accurate physical velocities of $v_{3}(z, t)$. The MATLAB simulations of $u_{1}(z, t)$, $u_{2}(z, t)$ and $\mathcal{W}(t)$ for different fixed $z$ or $t$ values are shown in Figs. 6, 7, 8, 9 and 10, while the Mathematica plots of $v_{3}(z, t)$ for different fixed $z$ or $t$ are shown in Figs. 11 and 12 , respectively. All simulations are dimensionless.

\section{Acknowledgements}

We acknowledge NWO and NDNS+ for the funding leading to this manuscript.

\section{Funding}

This work was funded by the Netherlands Organization for Scientific Research (NWO) under contract no. NWO-MPE 657.000.004. Moreover, we acknowledge the NWO cluster Nonlinear Dynamics in Natural Systems (NDNS+) for funding a research stay of AJV at Karlstads University to visit AM. These funding bodies had no roles in the design of the study and collection, analysis, and interpretation of data and in writing.

\section{Availability of data and materials}

The simulation codes and simulation data will become available at an open repository, when this paper is accepted.

\section{Authors' contributions}

The contributions of the authors are as followed: AJV 50\%, AM 10\%, FvdV 40\%. All authors read and approved the final manuscript.

\section{Authors' information}

AJV is a PhD student at both Eindhoven University of Technology in the Netherlands, and Karlstads Universitet, Sweden. AM is a full professor at Karlstads Universitet. FvdV is a emeritus professor at Eindhoven University of Technology.

\section{Ethics approval and consent to participate}

The authors declare that the research presented in this manuscript complies with the ethics guidelines of their respective institutions. The research presented in this manuscript is devoid of participation by individuals.

\section{Consent for publication}

The authors declare that the research presented in this manuscript is devoid of personal data of individuals.

\section{Competing interests}

The authors declare that they have no competing interests.

\section{Publisher's Note}

Springer Nature remains neutral with regard to jurisdictional claims in published maps and institutional affiliations.

Received: 17 May 2018 Revised: 27 July 2018 Accepted: 7 August 2018 Published online: 31 August 2018

\section{References}

1. Ali, G, Furuholt, $V$, Natalini, R, Torcicollo, I: A mathematical model of sulphite chemical aggression of limestones with high permeability. part ii: Numerical approximation. Transp. Porous. Media. 69(2), 175-188 (2007)

2. Arab, N, Zemskov, EP, Muntean, A, Fatima, T: Homogenization of a reaction-diffusion system modeling sulfate corrosion of concrete in locally periodic perforated domains. J. Engrg. Math. 69(2), 261-276 (2011)

3. Basista, M, Weglewski, W: Micromechanical modelling of sulphate corrosion in concrete: influence of ettringite forming reaction. Theoret. Appl. Mech. 35(1-3), 29-52 (2008)

4. Böhm, M., Devinny, J, Jahani, F, Rosen, G: On a moving-boundary system modeling corrosion in sewer pipes. Appl. Math. Comput. 92, 247-269 (1998)

5. Bouchelaghem, F: A numerical and analytical study on calcite dissolution and gypsum precipitation. Appl. Math. Model. 34, 467-480 (2010)

6. Bowen, RM: Incompressible porous media models by use of the theory of mixtures. Int. J. Engng. Sci. 18, 1129-1148 (1980)

7. Bowen, RM: Continuum Physics. vol. 3(Eringen, AC, ed.) Academic Press, New York (1976). Chap. 1. Theory of Mixtures

8. Bulíček, M, Málek, J, Rajagopal, R: On Kelvin-Voigt model and its generalizations (2010). Technical report, Nečas Center for Mathematical Modeling, Mathematical Institute, Charles University, Prague, Czech Republic 
9. Cahn, RW, Haasen, P, Kramer, EJ: Materials Science and Technology - A Comprehensive Treatment. Corrosion and Environmental Degradation, vol. 1. WILEY-VCH, Chichester (2000). Volume Editor: Michael Schütze

10. Chalupecky, V, Fatima, T, Muntean, A, Kruschwitz, J: Macroscopic corrosion front computations of sulfate attack in sewer pipes based on a micro-macro reaction-diffusion model. In: of Mathematics for Industry, FI (ed.) Multiscale Mathematics: Hierarchy of Collective Phenomena and Interrelations Between Hierarchical Structures. COE Lecture Note Series, pp. 22-31. Kyushu University, Japan, (2011). Collaborate Research Meeting of Institute of Mathematics for Industry \& Mext Collaborative Workshop of Mathematics and Mathematical Sciences with Various Sciences and Industrial Technologies, Fukuoka, Japan, December 8-11, 2011

11. Claisse, PA, et al.: In situ measurement of the intrinsic permeability of concrete. Mag. Concr. Res. 55, 125-132 (2003)

12. Clarelli, F, Fasano, A, Natalini, R: Mathematics and monument conservation: free boundary models of marble sulfation. SIAM J. Appl. Math. 69(1), 149-168 (2008)

13. During, EDD: Corrosion Atlas - A Collection of Illustrated Case Histories, 3rd exp. rev. edn. Elsevier, Amsterdam (1997)

14. Elsener, B: Materials Science and Technology - A Comprehensive Treatment. Corrosion and Environmental Degradation, vol. 2(Cahn, RW, Haasen, P, Kramer, EJ, eds.) WILEY-VCH, Chichester (2000). Chap. 8. Corrosion of Steel in Concrete. Volume Editor: Michael Schütze

15. Fasullo, GT: Sulphuric Acid: Use \& Handling. McGraw Hill, New York (1965)

16. Fatima, T, Muntean, A: Sulfate attack in sewer pipes: Derivation of a concrete corrosion model via two-scale convergence. Nonlinear Anal. Real World Appl. 15, 326-344 (2014)

17. Fusi, L, Farina, A, Primicerio, M: A free boundary problem for $\mathrm{CaCO}_{3}$ neutralization of acid waters. Nonlinear Anal. Real World Appl. 15, 42-50 (2014). https://doi.org/10.1016/j.nonrwa.2013.05.004

18. Fusi, L, Primicerio, M, Monti, A: A model for calcium carbonate neutralization in the presence of armoring. Appl. Math. Modell. 39, 348-362 (2015). https://doi.org/10.1016/j.apm.2014.05.037

19. Gu, J-D, Ford, TE, Mitchell, R: Uhlig's Corrosion Handbook, 3rd edn(Winston Revie, R, ed.) Wiley, Hoboken (2011). Chap. 32. Microbial Corrosion of Concrete

20. Haynes, WM, Lide, DR, Bruno, TJ: CRC Handbook of Chemistry and Physics. 97th edition edn. CRC Press, Boca Raton (2017)

21. Holmes, MH: Introduction to Perturbation Methods. 2nd edn. Texts in Applied Mathematics. vol. 20. Springer, Berlin (2013)

22. Kierzenka, J, Shampine, LF: A BVP solver based on residual control and the MATLAB PSE. ACM Trans. Math. Software. 27(3), 299-316 (2001)

23. Kierzenka, J, Shampine, LF: A BVP solver that controls residual and error. J. Num. Anal., Indus. \& Appl. Math. 3(1-2), 27-41 (2008)

24. Lafhaj, Z, et al.: Experimental determination of intrinsic permeability of limestone and concrete: Comparison between in situ and laboratory results. Build. Environ. 42, 3042-3050 (2007)

25. Meyers, MA, Chawla, K: Mechanical Behavior of Materials. 2nd edn. Cambridge University Press, Cambridge (1999)

26. Monteiro, PJM: Interfacial Transition Zone in Concrete. 1st edn. RILEM Report(Maso, JC, ed.) E \& FN SPON, London (1996). Chap. 4. Mechanical modelling of the transition zone. State-of-the-Art Report prepared by RILEM Technical Committee 108-1CC, Interfaces in Cementitious Composites

27. Morro, A: Diffusion in mixtures of reacting thermoelastic solids. J. Elast. 123, 59-84 (2015)

28. Nikolopoulos, CV: A mushy region in concrete corrosion. Appl. Math. Model. 34, 4012-4030 (2010)

29. Nikolopoulos, CV: Macroscopic models for a mushy region in concrete corrosion. J. Engrg. Math. 91(1), 143-163 (2014)

30. Nikolopoulos, CV: Mathematical modelling of a mushy region formation during sulphation of calcium carbonate. Netw. Heterog. Media. 9(4), 635-654 (2014)

31. Ortiz, M, Popov, EP: Plain concrete as a composite material. Mech. Mater. 1,139-150 (1982)

32. Plachy, T, Tesarek, P, Padevet, P, Polak, M: Determination of Young's modulus of gypsum blocks using two different experimental methods. In: Bulucea, CA (ed.) Recent Advances in Applied and Theoretical Mechanics. WSEAS Press, (2009). www.wseas.org. 5th WSEAS Int. Conf. Appl. Theor. Mech.
33. Rendell, F, Jauberthie, R, Grantham, M: Deteriorated Concrete. Thomas Telford Publishing, London (2002)

34. Rice, JA: Mathematical Statistics and Data Analysis. 3rd edn. Duxbury Advanced Series. Thomson Brooks/Cole, Pacific Grove (2007). International Student Edition

35. Sand, W: Materials Science and Technology - A Comprehensive Treatment. Corrosion and Environmental Degradation, vol. 1(Cahn, RW, Haasen, P, Kramer, EJ, eds.) WILEY-VCH, Chichester (2000). Chap. 4. Microbial Corrosion. Volume Editor: Michael Schütze

36. Shampine, LF, Reichelt, MW, Kierzenka, J: Solving boundary value problems for ordinary differential equations in matlab with bvp $4 \mathrm{C}$. Technical report, Math. Dept., SMU, Dallas (2000). The tutorial and programs are available at http://www.mathworks.com/bvp_tutorial

37. Taylor, HFW: Cement Chemistry. 2nd edn. Thomas Telford Publishing, London (1997)

38. Trethewey, KR, Chamberlain, J: Corrosion for Science \& Engineering. 2nd edn. Longman Group, Harlow (1995)

39. Verdink Jr., ED: Economics of Corrosion. In: Winston Revie, R (ed.) Uhlig's Corrosion Handbook, 3rd edn. Wiley, Hoboken, (2011)

40. Vromans, AJ: A pseudoparabolic reaction-diffusion-mechanics system: modeling, analysis and simulation. Licentiate thesis, Karlstad University (2018)

41. Wittmann, HF: Estimation of the modulus of elasticity of calcium hydroxide. Cem. Concr. Compos. 16, 971-972 (1986)

\section{Submit your manuscript to a SpringerOpen ${ }^{\circ}$ journal and benefit from:}

- Convenient online submission

- Rigorous peer review

- Open access: articles freely available online

- High visibility within the field

- Retaining the copyright to your article

Submit your next manuscript at $\$$ springeropen.com 